\title{
Optimization-based minimum-cost seismic retrofitting of hysteretic frames with nonlinear fluid viscous dampers
}

\author{
Pollini, Nicolo; Lavan, Oren; Amir, Oded
}

Published in:

Earthquake Engineering and Structural Dynamics

Link to article, DOI:

10.1002/eqe.3118

Publication date:

2018

Document Version

Peer reviewed version

Link back to DTU Orbit

Citation (APA):

Pollini, N., Lavan, O., \& Amir, O. (2018). Optimization-based minimum-cost seismic retrofitting of hysteretic frames with nonlinear fluid viscous dampers. Earthquake Engineering and Structural Dynamics, 47(15), 29853005. https://doi.org/10.1002/eqe.3118

\section{General rights}

Copyright and moral rights for the publications made accessible in the public portal are retained by the authors and/or other copyright owners and it is a condition of accessing publications that users recognise and abide by the legal requirements associated with these rights.

- Users may download and print one copy of any publication from the public portal for the purpose of private study or research.

- You may not further distribute the material or use it for any profit-making activity or commercial gain

- You may freely distribute the URL identifying the publication in the public portal 


\title{
Optimization-based minimum-cost seismic retrofitting of hysteretic frames with nonlinear fluid viscous dampers
}

\author{
Nicolò Pollini*1, Oren Lavan ${ }^{\dagger 2}$ and Oded Amir ${ }^{\ddagger 2}$ \\ ${ }^{1}$ Department of Mechanical Engineering, Technical University of Denmark, Kgs Lyngby, Denmark \\ ${ }^{2}$ Faculty of Civil and Environmental Engineering, Technion - Israel Institute of Technology, Haifa, Israel
}

This is an unformatted version of the paper, published online in Earthquake Engineering \& Structural Dynamics, September 2018.

\begin{abstract}
In this paper we discuss an optimization-based approach for minimum-cost seismic retrofitting of hysteretic frames with nonlinear fluid viscous dampers. The proposed approach accounts also for for moment-axial interaction in the structural elements, to consider a more realistic coupling between added dampers and retrofitted structure. The design variables of the problem are the damping coefficients of the dampers. Indirectly, the design involves also the stiffness coefficients of the supporting braces. In the optimization analysis, we minimize a realistic retrofitting cost function with constraints on inter-story drifts under a suite of ground motion records. The cost function includes costs related to the topological and mechanical properties of the dampers' designs. The structure is modeled with a mixed finite element approach, where the hysteretic behavior is defined at the beams' and columns' cross-sections level. We consider damper-brace elements with a visco-elastic behavior characterized by the Maxwell model. The dampers' viscous behavior is defined by a fractional power law. Promising results obtained for a two-story, a nine-story, and a twenty-story 2-D frames are presented and discussed.
\end{abstract}

Keywords: seismic retrofitting, optimization, hysteretic structures, viscous dampers, Maxwell model.

\section{Introduction}

Recently, with the revival of tall building construction, new research efforts have been directed towards the study of the seismic performance of high-rise buildings. One of the outcome has been the Tall Building Initiative (TBI) of the Pacific Earthquake Engineering Research Center (PEER). The phase $I$ of the TBI has been focusing on the development of background documents and guidelines for the construction of new high-rise buildings [1, 2]. A more sensitive topic seems to be the seismic performance of existing tall building that were constructed when the knowledge and the understanding regarding the construction of high-rise structures under seismic hazards was much less advanced. After the assessment of the condition of the structures damaged during the 1994 Northridge earthquake it became clear that many of the existing high-rise structures where not safe enough in the eventuality of an earthquake. In fact, in nearly two hundred steel moment resisting frames (SMRFs) with pre-Northridge connections, brittle beam-to-column connection failures were encountered [3]. As a result, phase $I I$ of the TBI focuses on the study of the seismic performance and feasible retrofitting solutions for this type of structures.

A number of different approaches for the seismic retrofitting of tall buildings are available [4]. One approach consists of increasing the deformation capacity of existing connections. Nevertheless, it was noted that with ductile connections and columns' splices, large inter-story drifts demand and residual displacements were encountered [4]. Hence, the cost required to retrofit the ductile connections in the post-earthquake phase makes this type of approach economically prohibitive. The search for a more

\footnotetext{
*nipol@mek.dtu.dk, nicolo@technion.ac.il

$\dagger$ lavan@technion.ac.il

‡odedamir@technion.ac.il
} 
economical retrofitting strategy able to reduce deformation demands, and the tendency of the structure to form weak-story mechanisms led to the seismic retrofitting of tall buildings with fluid viscous dampers [5]. The use of passive energy dissipation systems is gaining much attention from both researchers and practitioners, and the reader is referred to the comprehensive textbooks for more details [6, 7]. The design of added fluid viscous dampers includes several key steps, such as the selection of the locations in the structure for dampers within given architectural constraints, the sizing of the dampers (typically done by selecting their damping coefficients), and the minimization of the associated cost. Identifying economical dampers' distributions and sizes that improve the behavior of a given structure to a desired performance level is not intuitive. Moreover, different configurations of dampers may lead to very different behaviors and final costs of the retrofitted structure [8].

A promising direction for the solution of this type of seismic retrofitting problem, seems to be the development of approaches for the distribution and sizing of viscous dampers based on optimization. Several authors focused on the optimization-based seismic retrofitting of linear structures with linear viscous dampers [9, 10, 11, 12, 13, 14, 15, 16, 17. However, there are structures whose behavior can not be expected to be linear in the eventuality of severe seismic events, even if a reasonable amount of damping is added to the structure. This category could include, for example, irregular structures and tall buildings. The use of linear analysis tools may fail to give a good estimate of the structural response in these cases. This will also be demonstrated through the examples in this paper. Hence, nonlinear analysis tools are required. Nonlinear analysis tools are also needed when a more detailed study of the structural response due to the coupling of an added damping system is required. The use of nonlinear viscous dampers, in fact, if on the one hand may reduce inter-story drifts and story accelerations, on the other hand may increase further the axial forces in the columns and hence reduce the columns' capacity for bending moments. This may be particularly relevant in the case of tall buildings, where the columns at the bottom stories may undergo large compression states. With respect to this, there are several contributions of authors that focused on the retrofitting of nonlinear structures with linear dampers [18, 19, 20, 21, 22, 23, 24, 25].

From the dampers' modeling side, when viscous dampers are modeled as pure dashpots their supporting braces are implicitly assumed as infinitely stiff. This may be an inadequate assumption since braces have an upper limit in terms of axial stiffness. Recently, it was also observed that considering a more realistic finite stiffness of the brace would significantly affect the damper-brace mechanical behavior [26]. Moreover, nowadays viscous dampers with a nonlinear force-velocity behavior are considered by researchers [27, 28, 29, 30, 31, engineers [32], and producers [33]. Nonlinear dampers also proved to be able to achieve the same level of response reduction of a structure retrofitted with linear viscous dampers, but with smaller forces in the nonlinear dampers case [34. And since the dampers' manufacturing cost depends on their peak guaranteed force [35], using viscous dampers with a nonlinear behavior may have also economical advantages. Motivated by these considerations, Pollini et al. [36] considered the minimumcost seismic retrofitting of 3-D structures equipped with nonlinear viscous dampers. The damper-brace elements were modeled through the Maxwell model for visco-elasticity [16], and the dampers-brace elements were placed and sized with a gradient-based algorithm while minimizing a realistic cost function. Nonetheless, the retrofitted structures were assumed to remain elastic. Recently, Gidaris and Taflanidis [37] presented an optimization-based approach for the minimization of the life-cycle cost for nonlinear structures with nonlinear dampers. This indeed presents another step towards a more realistic structural model. Nonetheless, zero order methods were used for the optimization. Using such methods, the computational effort involved is usually very large. This highlights the need for a computationally efficient method for the optimal seismic retrofitting of nonlinear structures using nonlinear dampers.

The approach presented and discussed in this paper constitutes an additional contribution to the retrofitting problem of nonlinear structures with nonlinear fluid viscous dampers, through a practical and computationally efficient optimization-based approach. The proposed approach originates from previous work of the authors, where the minimum-cost seismic retrofitting of linear structures with linear [17] and nonlinear [36] fluid viscous dampers was considered. However, in the eventuality of more severe seismic excitations, a nonlinear structural response should be considered. Thus, herein we will consider beam-column elements with a nonlinear hysteretic behavior defined at the element sections level. In the case of tall buildings, the columns at the lowers stories may undergo large compression states during an earthquake. Additionally, the presence of fluid viscous dampers in the structure may further increase the 
axial compression in the columns, and as a consequence their moment capacity may be reduced. Hence, we will include also a simplified interaction model between axial force and bending moment in the elements. From a computational point of view, the analysis of nonlinear structures with nonlinear dampers represents the most demanding analysis problem. In turn, the complex behavior of such mechanical systems, and in particular the M-N interaction in the columns, also results in a highly nonlinear optimization problem. Additionally, large scale structures are characterized by large numbers of design variables such as those related to the dampers' size and placement in the structure. To avoid a prohibitive computational effort required by the optimization process, it is desirable to rely on a gradient-based optimization approach. In particular, we will consider damper-brace elements made of a spring and a nonlinear dashpot in series. In this way it will be possible to account for the stiffening contribution of the braces and of the dampers, and for the damping property of the viscous dampers. As in Pollini et al. [36], here too a realistic retrofitting cost formulation is minimized. In each potential location, the optimization algorithm determines if a damper is needed or not. If a damper is needed, it is also determined which size-group it belongs to. Each size-group is characterized by nonlinear damper-brace elements with the same mechanical properties. The size-groups are limited in number to achieve final practical designs, but their properties are not predefined and are optimized simultaneously within a single problem formulation. Inter-story drifts are constrained to allowable values. These are evaluated with nonlinear time-history analyses considering realistic ground motions. Initially, to create benchmark results for comparison, the problem is formulated as a mixed-integer optimization problem, and is solved with a Genetic Algorithm (GA). Subsequently, the initial mixed-integer problem formulation is translated into a continuous one. This allows the solution of the nonlinear programming problem through a gradient-based Sequential Linear Programming approach (SLP), which reduces significantly the computational cost required for its solution. Final practical designs are achieved by means of material interpolation functions. It should be noted that with none of the two optimization algorithms considered (i.e. GA and SLP) there is a formal guarantee that the final design solution will be also the global minima of the optimization problem at hand.

The remainder of the article is organized as follows: In Sec. 2 we present the governing equations of the problem. The mixed-integer programming problem formulation is introduced in Sec. 3, with details on the parametrization of the design variables, the performance index, and the cost function considered. In Sec. 4 the mixed-integer programming problem is approximated with a continuous optimization problem formulation. The details for this approximation are given in dedicated sections. Next, in Sec. 5 the results regarding the optimization of a two-story, a nine-story, and a twenty-story frame are presented. In Sec. 6] some final considerations and conclusions are drawn.

\section{Governing equations}

In this section, we discuss the models considered for the structural elements and the nonlinear fluid viscous dampers. In particular, we first present the force-based finite element model adopted for the beam elements, and the model used for the characterization of the damper-brace behavior. We conclude this section by presenting the system of equations for the dynamic equilibrium of the structural systems considered, with a brief overview of the algorithm adopted for the response analysis.

\subsection{Beam finite element: A mixed formulation}

We consider a Euler-Bernoulli beam element type, which is appropriate for slender beam-column elements. The finite element formulation has a mixed form [38], as presented by Spacone et al. in Reference 39] and Reference [40. In particular, the global equilibrium equations of the structure are written through the direct stiffness method (i.e. the degrees of freedom are the nodal displacements) which allows to sum up the contributions of all the elements for the assembly of the structure stiffness matrix and resisting forces. However, at the element level in the mixed formulation considered herein forces are interpolated rather than displacements (i.e. the degrees of freedom are the nodal forces). The forces' interpolation is exact, and it leads to a consistent state determination which does not add additional intrinsic approximations. However, the state determination of the mixed finite element approach considered herein is more complex than that of more traditional displacement-based finite element approaches, and it is 


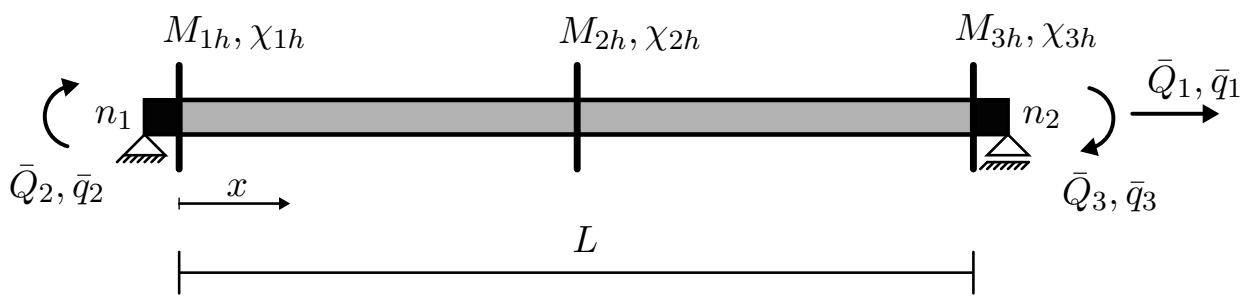

(a) Force-based beam element $h$ with three control sections (i.e. Gauss-Lobatto points). The internal forces in each section are obtained by interpolating the nodal forces

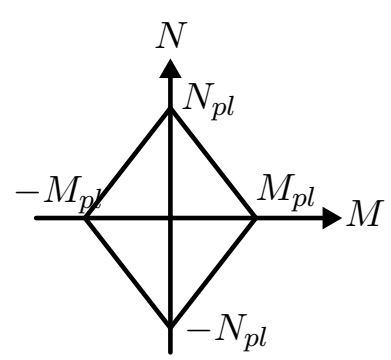

(b) Simplified axial force and bending moment domain of interaction considered

Figure 1: Structural model: beam finite element, and element domain of interaction.

governed by nonlinear equations. Hence, the state determination is performed through an additional iterative procedure.

\subsubsection{Force-based beam element}

We consider the element presented in Fig. 1(a). The independent nodal forces will be denoted with $\overline{\mathbf{Q}}$, and the independent nodal displacements with $\overline{\mathbf{q}}$. They act on a system that can be seen as the element "without rigid body modes".

In our formulation we consider a simplified linear elastic axial behavior defined at the element level. With reference to a beam element $h$ without rigid body modes we have that:

$$
\bar{Q}_{1, h}=\frac{E A_{h}}{L_{h}} \bar{q}_{1, h}
$$

where $\bar{Q}_{1, h}$ is the axial force in the element without rigid body modes, $E$ is the Young's modulus (e.g. for steel $E=200 \mathrm{GPa}$ ), $A_{h}$ is the section area of the element, $L_{h}$ is the length of the element, and $\bar{q}_{1, h}$ its axial elongation. We will consider a nonlinear hysteretic bending behavior of the structure, defined at the beam sections level. The potential formation of local instabilities due to local buckling as well as instabilities due to lateral torsional buckling of the elements will not be accounted for. In particular, we will consider a fixed number of control sections where the bending behavior is evaluated during the dynamic analysis. The behavior of the control sections is defined in terms of a differential moment-curvature (i.e. $M-\chi$ ) relation. In this work we considered a hysteretic model developed by Sivaselvan and Reinhorn [41], even though any moment-curvature relation can be considered as long as it is formulated with a differentiable form. The model considered herein is the following:

$$
\dot{M}_{h}^{k}=\left\{a E I_{h}+(1-a) E I_{h}\left[1-0.5\left|\frac{M_{h}^{k, *}}{M_{y, h}^{*}}\right|^{N}\left(\operatorname{sgn}\left(M_{h}^{k, *} \dot{\chi}_{h}^{k}\right)+1\right)\right]\right\} \dot{\chi}_{h}^{k}
$$

with:

$$
M_{h}^{k, *}=M_{h}^{k}-a E I_{h} \chi_{h}^{k}, \quad M_{y, h}^{*}=(1-a) M_{y, h} .
$$

In Eq. (2) and Eq. (3) $\dot{M}_{h}^{k}$ and $\dot{\chi}_{h}^{k}$ are the time derivatives of the moment $M_{h}^{k}$ and curvature $\chi_{h}^{k}$ of the section $k$ of the element $h ; I_{h}$ is the section inertia of the element; $M_{y, h}$ is the section yielding moment calculated through the product of the yielding stress $f_{y}$ and the plastic section modulus $W_{p l} ; a$ is the ratio between the residual secondary stiffness in the post yielding range, and the initial elastic stiffness (e.g. $a=2 \%) ; \operatorname{sgn}(\cdot)$ is the sign function. The moment in one of the control sections is calculated by interpolating the end moments.

\subsubsection{N-M interaction}

We consider a simplified coupled behavior between the axial force and the bending moment in each element. In particular, in a time-step $i$ the current value of the yielding moment of a generic element $h$, 
$M_{y, i}^{h}$, depends on the value of the axial force in the pevious time-step, $N_{i-1}^{h}$. This type of interaction was inspired by the one adopted in the structural analysis software Ruaumoko [42]. The interaction can be formulated as follows:

$$
M_{y, i}^{h}=\left|-\left(\frac{M_{p l}^{h}}{N_{p l}^{h}}\right)\right| N_{i-1}^{h}\left|+M_{p l}^{h}\right| .
$$

Fig. 1(b) shows the graphical representation of the $N-M$ interaction domain. As it can be seen from Eq. (4) and its representation in Fig. 1(b) the interaction model that we consider is symmetric with respect to the axial force and the bending moment. This type of behavior could represent the one for steel elements. However, this should not be seen as a restriction or a limitation. In fact the methodology discussed here can accommodate other types of models for the $N-M$ interaction, as long as they are formulated with differentiable mathematical formulations. It should be noted that the formulation of Eq. (4) is differentiable except when the argument of the absolute value functions (i.e. $|\cdot|$ ) is equal to zero. However, since we will calculate the gradients of the functions involved considering the discretized version of the problem formulation, the arguments of the absolute value functions will be most likely never exactly equal to zero. Thus, from a numerical point of view, we can consider Eq. (4) differentiable.

\subsubsection{Element state determination}

In a mixed formulation, the quantities in input and output in the state determination are the same as in a displacement-based formulation. That is, in input we have the element nodal displacements, $\mathbf{q}$, and in output we have the nodal forces and the element stiffness, $\mathbf{Q}$ and $\mathbf{K}$. The state formulation of a mixed finite element formulation relies on the exact interpolation of the nodal forces in order to calculate the generalized stresses (i.e. $N(x), M(x)$ ) along the beam axis. Since the quantities in input (i.e. q) are not those actually interpolated (i.e. Q), there is the so called lack-of-fit that causes the state determination of the mixed finite element formulation adopted herein to be more complicated and governed by a set of nonlinear equations. As a consequence, the state determination is not straight forward as in the displacement-based formulation, and it requires an iterative procedure. For more details regarding the element state determination considered in this paper the reader is referred to Spacone et al. [39]

\subsection{Damper-brace element formulation}

We model the dampers with the Maxwell's model considering a spring and a dashpot in series, as shown in Fig. 2. The spring accounts for the stiffening property of the damper and its supporting member, and the dashpot for its viscous property.

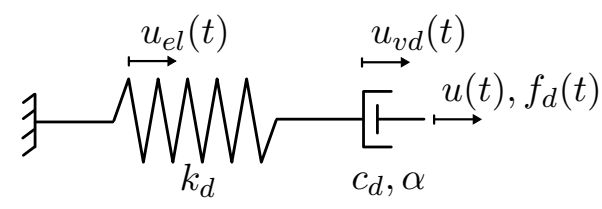

Figure 2: Nonlinear viscous damper modeled with the Maxwell's model.

The first order differential equation that defines the behavior of the damper-brace element is the following:

$$
\dot{f}_{d}(t)=k_{d}\left(\dot{u}(t)-\operatorname{sgn}\left(f_{d}(t)\right)\left(\frac{\left|f_{d}(t)\right|}{c_{d}}\right)^{\frac{1}{\alpha}}\right)
$$

where: $k_{d}$ accounts for the stiffness of the brace and of the damper; $\dot{u}(t)$ is the relative velocity between the damper elements ends at time $t ; \operatorname{sgn}()$ is the sign function; $f_{d}(t)$ is the force in the damper at time $t ; c_{d}$ the damping coefficient of the dashpot; and $\alpha$ the exponent of the fractional power law that defines the nonlinear force-velocity behavior of the dashpot. In this work we consider a fixed ratio $\rho$ between the stiffness coefficient $k_{d}$ and the damping coefficient $c_{d}$. This ratio can affect how the damper element will behave and the ease with which its first order differential equation can be approximated numerically. For the tuning procedure of the parameter $\rho$ we relied on the procedure discussed in Pollini et al. [36] During the nonlinear time-history structural analysis, the response of each damper in each time-step is 
approximated with a 4-th order Runge-Kutta method as suggested by Kasai and Oohara [43. A similar approach has been used in 44 and by the authors in Reference [45].

\subsubsection{Equations of motion}

The equations for the dynamic equilibrium of the structural systems considered are the following:

$$
\mathbf{M} \ddot{\mathbf{u}}(t)+\mathbf{C}_{s} \dot{\mathbf{u}}(t)+\mathbf{F}_{s}(t)+\mathbf{F}_{d}(t)=-\mathbf{M e} a_{g}(t), \quad \forall t=t_{0}, \ldots, t_{f}
$$

where: $\mathbf{M}$ is the mass matrix; $\mathbf{C}_{s}$ is the inherent damping matrix; $\mathbf{F}_{s}(t)$ is the vector of the resisting forces of the structural elements at time $t ; \mathbf{F}_{d}(t)$ is the vector of the resisting forces of the dampers at time $t$; $\mathbf{e}$ is the influence vector and it represents the displacements of the masses resulting from static application of a unit ground displacement. Essentially, it distributes the acceleration over the degrees of freedom of the structure affected by the ground motion; $a_{g}(t)$ the ground acceleration record as a function of time; $\ddot{\mathbf{u}}(t), \dot{\mathbf{u}}(t), \mathbf{u}(t)$ are the acceleration, velocity and displacement vectors of the degrees of freedom of the system at time $t$. The initial conditions are:

$$
\mathbf{u}(0)=\mathbf{u}_{0}, \quad \dot{\mathbf{u}}(0)=0, \quad \mathbf{F}_{s}(0)=\mathbf{F}_{s, 0}, \quad \mathbf{F}_{d}(0)=0
$$

and they are evaluated with a static analysis considering the gravity loads.

To build the mass matrix $\mathbf{M}$ we consider two concentrated masses at the ends of each beam element. These masses account for the structural weight, and the loads carried by the structure. The matrix $\mathbf{C}_{s}$ is the inherent Rayleigh damping matrix, and it is built considering the initial stiffness matrix. It should be noted that in the case of inelastic dynamic analysis, the Rayleigh damping matrix may lead to un-realistic damping forces and moments on the onset of yielding [47. However, when added dampers are used, as in the work discussed herein, this source of inconsistency may be disregarded and the Rayleigh damping matrix can be considered [48]. For the integration scheme we rely on a time stepping algorithm based on the Newmark- $\beta$ method: in particular we relied on the Newmark- $\beta$ average acceleration method.

The algorithm for the solution of the nonlinear dynamic equilibrium equations is subdivided into three nested loops: The outer is the loop denoted by the index $k$, and it follows the time discretization of the problem. In each step, we apply the load $\mathbf{P}_{k}=-\mathbf{M e} a_{g, k}$ on the structure and we want to calculate the response vectors of the structure and the associated resisting forces of the elements (i.e. $\mathbf{u}_{k}, \dot{\mathbf{u}}_{k}, \ddot{\mathbf{u}}_{k}$, $\mathbf{F}_{s, k}$, and $\mathbf{F}_{d, k}$ ); The second, loop denoted by the index $i$, contains the state determination of the whole structure. Essentially, in this loop the goal is to calculate the displacements, velocities and accelerations of the structure and the resisting forces caused by the applied loads $\mathbf{P}_{k}\left(\right.$ i.e. $\mathbf{u}_{k}^{i}, \dot{\mathbf{u}}_{k}^{i}, \ddot{\mathbf{u}}_{k}^{i}, \mathbf{F}_{s, k}^{i}$, and $\mathbf{F}_{d, k}^{i}$ ). In the algorithm used in this work, this is achieved by means of the Newton-Raphson method. Moreover, it is in this loop that the vector of the dampers' forces is approximated with a 4-th order Runge-Kutta approximation [49, 45]; The inner loop is denoted by the index $j$. In this loop the state determination for each element is contained. The input are the nodal displacements and velocities of each element, and the goal is to calculate a compatible distribution of curvatures, associated to an equilibrated distribution of moments. The output are the nodal forces and the stiffness of each element. These quantities are then assembled to verify the convergence to an equilibrium state in the loop denoted by $i$. Also in this loop, the state determination for the elements is performed with the Newton-Raphson method. For more details on the element state determination considered the reader is referred to Spacone et al. [39]

\section{Optimization problem formulation}

In this section, we present the optimization problem for the seismic retrofitting of frames with fluid viscous dampers. Important aspects of the problem formulation that will be discussed are: The parametrization of the design variables of the problem (i.e. the dampers' damping coefficients), which includes both discrete and continuous variables that allow to identify final discrete and practical designs without predefining any aspect of the problem beforehand; The retrofitting cost function which will be minimized in the optimization analysis; And last, the constrained structural performance indices. 


\subsection{Parametrization of the design variables}

The retrofitting methodology discussed herein consists of allocating and sizing nonlinear fluid viscous dampers and their supporting braces in $N_{d}$ predefined potential locations of a given frame. In each location we allow as many as two damper-brace elements. Either one of two available sizes can be assigned to the damper-brace elements. The two available size are also variables of the problem. Thus, the final designs are characterized by two size-groups, where for size-group we intend a group of dampers with the same mechanical properties. The size is defined by a damping coefficient. Therefore, we want to determine the damping coefficients $c_{d, i}$ of each $i$-th damper, that are collected in the vector $\mathbf{c}_{d}$. Through the ratio $\rho$, the design is indirectly extended also to the associated stiffness coefficients $k_{d, i}$, collected in the vector $\mathbf{k}_{d}$. The vector of damping coefficients is:

$$
\mathbf{c}_{d}=\bar{c}_{d} \mathcal{D}\left(\mathbf{x}_{1}\right)\left(y_{1} \mathbf{1}+\left(y_{2}-y_{1}\right) \mathbf{x}_{2}\right), \quad \mathbf{k}_{d}=\rho \mathbf{c}_{d}
$$

In Eq. (8), $\mathcal{D}(\cdot)$ is the diagonal matrix operator that transforms a vector into a diagonal matrix (similarly to the diag $(\cdot)$ Matlab function). The parameter $\bar{c}_{d}$ represents the maximum damping coefficient available, and it is predefined. The vector $\mathbf{1}$ has unit entries and size $2 N_{d} \times 1$. The vector $\mathbf{x}_{1}$ has binary entries, and it describes the existence of a damper in each potential locations: A value of zero in the $i$-th entry of the vector means that the $i$-th damper does not exist, while a value of one that the damper exists. Also $\mathbf{x}_{2}$ is a vector with binary entries, and it represents the association of each existing damper to one of the two available size-groups. In the case of $x_{2, i}$ equal to zero, the $i$-th damper belongs to the first size-group. In the case of $x_{2, i}$ equal to one, the $i$-th damper belongs to the second size-group. The dimensions of the vectors $\mathbf{c}_{d}, \mathbf{x}_{1}$, and $\mathbf{x}_{2}$ are $2 N_{d} \times 1$. The two available damping coefficients that characterize the two size-groups are:

$$
\bar{c}_{1}=\bar{c}_{d} y_{1} ; \quad \bar{c}_{2}=\bar{c}_{d} y_{2} .
$$

In Eq. (9), $y_{1}$ and $y_{2}$ are two continuous design variables that scale the maximum available damping coefficient $\bar{c}_{d}$. The column vector $\mathbf{x}$ collects all the variables of the problem: $\mathbf{x}^{T}=\left[\mathbf{x}_{1}^{T}, \mathbf{x}_{2}^{T}, y_{1}, y_{2}\right]$.

\subsection{Objective cost function}

One of the main objectives of the work herein discussed is to identify minimum-cost retrofitting designs. Therefore, the goal is to minimize a realistic formulation of the costs associated to the retrofitting designs. In particular, the cost function considered, which is the objective function in the optimization problem, is composed of three components: $J=J_{l}+J_{m}+J_{p}$. The first component was presented in Reference [17] and here it has been adjusted to accommodate a slightly different problem formulation. The second component was presented first in Reference [36]. The third cost component is common to both the works just mentioned.

The first component $J_{l}$ considers the number of locations in which dampers are installed. This cost includes all the aspects related to the preparation of the structure for the damper installation and the architectural constraint that this installation will represent. Moreover, in case of retrofitting, the removal of existing nonstructural components can also be considered. We allow the algorithm to allocate as many as two dampers in each potential location. This cost component is formulated so that it will be more expensive to allocate a damper in an empty potential location than to allocate the same damper in a location where a damper already exists. This is because the location will be considered as already "opened" and the associated costs already covered. The first component of the cost is defined as follows:

$$
J_{l}=\mathbf{x}_{1}^{T} \mathbf{C}_{l}
$$

where $\mathbf{C}_{l}$ is a $2 N_{d} \times 1$ vector in which the $i$-th component is a cost component related to the $i$-th component of $\mathbf{x}_{1}$. The vector $\mathbf{C}_{l}$ is defined as follows:

$$
\begin{aligned}
& \mathbf{C}_{l}=\mathcal{D}\left(\mathbf{C}_{l 1}\right)\left[\begin{array}{lllll}
1 & 1 & \ldots & 0 & 0
\end{array}\right]^{T}+
\end{aligned}
$$

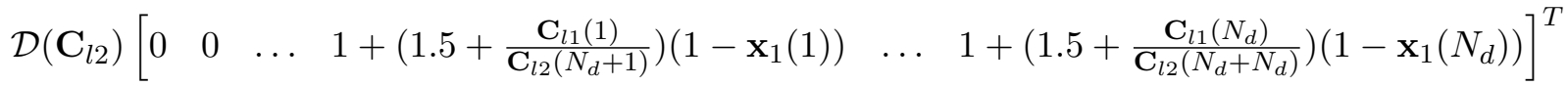


where $\mathbf{C}_{l 1}$ represents the specific cost of installation of the first damper in a potential location, its dimensions are $2 N_{d} \times 1$ and it has the first $N_{d}$ elements different from zero; $\mathbf{C}_{l 2}$ represents the cost for each potential location of adding the second damper assuming the first damper is already installed at that location, its dimensions are $2 N_{d} \times 1$ and it has the last $N_{d}$ elements different from zero. Both $\mathbf{C}_{l 1}$ and $\mathbf{C}_{l 2}$ are vectors defined by the user. Because $\mathbf{C}_{l 1}(k) \geq \mathbf{C}_{l 2}\left(k+N_{d}\right) \forall k$, the cost of installing the first damper in a potential location is larger than the cost of installing the second one, provided that the first damper is already installed. The vector that multiplies $\mathcal{D}\left(\mathbf{C}_{l 1}\right)$ has the first $N_{d}$ entries equal to one, and the remaining equal to zero. The vector that multiplies $\mathcal{D}\left(\mathbf{C}_{l 2}\right)$ has the first $N_{d}$ entries equal to zero, and the remaining $N_{d}$ are defined so that it will be more expensive to first allocate the second damper in an empty location than to allocate the first one in the same location: Referring to the $i$-th location, in the first case the cost will be $\mathbf{C}_{l 1}(i)+2.5 \times \mathbf{C}_{l 2}\left(i+N_{d}\right)$ while in the second case only $\mathbf{C}_{l 1}(i)$.

The second cost component, $J_{m}$, represents the manufacturing cost of the dampers. In principle, the manufacturing cost of viscous dampers depends on the peak stroke and on the square root of the peak force of the most loaded damper of each size-group [35. We assume, in fact, that all dampers of a specific size-group are designed so to have the same capacity. Since we are constraining inter-story drifts, also the peak stroke of the dampers is indirectly limited. As a consequence, it does not affect significantly the cost. Therefore, the manufacturing cost is defined as the square root of the peak force of the most loaded damper from each size-group, multiplied by the number of dampers of the corresponding size-group. Formally, it is written as follows:

$$
J_{m}=C_{m}\left\{\mathbf{x}_{1}^{T}\left(\mathbf{1}-\mathbf{x}_{2}\right)\left[\max _{i}\left(\hat{f}_{d 1, i}\right)\right]^{0.5}+\mathbf{x}_{1}^{T} \mathbf{x}_{2}\left[\max _{i}\left(\hat{f}_{d 2, i}\right)\right]^{0.5}\right\}
$$

where: $C_{m}$ is a coefficient used to homogenize the units of measure of $J_{m}$ to the units of measure of $J_{l}$ and $J_{p} ; \mathbf{x}_{1}^{T}\left(\mathbf{1}-\mathbf{x}_{2}\right)$ is the number of dampers of the first size-group; $\mathbf{x}_{1}^{T} \mathbf{x}_{2}$ is the number of dampers of the second size-group. Additionally:

$$
\begin{aligned}
& \hat{\mathbf{f}}_{d 1}=\left[\begin{array}{lllll}
\hat{f}_{d 1,1} & \ldots & \hat{f}_{d 1, i} & \ldots & \hat{f}_{d 1, N_{d}}
\end{array}\right]^{T}=\mathcal{D}\left(\mathbf{1}-\mathbf{x}_{2}\right) \hat{\mathbf{f}}_{d} \\
& \hat{\mathbf{f}}_{d 2}=\left[\begin{array}{lllll}
\hat{f}_{d 2,1} & \ldots & \hat{f}_{d 2, i} & \ldots & \hat{f}_{d 2, N_{d}}
\end{array}\right]^{T}=\mathcal{D}\left(\mathbf{x}_{2}\right) \hat{\mathbf{f}}_{d} \\
& \hat{\mathbf{f}}_{d}=\max _{t}\left(\left|\mathbf{f}_{d}(t)\right|\right)
\end{aligned}
$$

$\hat{\mathbf{f}}_{d}$ is the vector of the peak forces in time for all dampers; the vector $\hat{\mathbf{f}}_{d 1}$ has the components of $\hat{\mathbf{f}}_{d}$ which belong to dampers of the first size-group, while $\hat{\mathbf{f}}_{d 2}$ those of the second size-group.

Modern seismic codes require to test one damper prototype from each size-group to verify its forcevelocity behavior. As a results, we consider an additional cost component, $J_{p}$. This component is formulated so that the number of different size-groups of dampers actually used for retrofitting should be minimized:

$$
J_{p}=C_{p}\left[\mathcal{H}\left(\mathbf{x}_{1}^{T}\left(\mathbf{1}-\mathbf{x}_{2}\right)\right)+\mathcal{H}\left(\mathbf{x}_{1}^{T} \mathbf{x}_{2}\right)\right] ; \quad \mathcal{H}(x)=\left\{\begin{array}{l}
1 \text { for } x>0 \\
0 \text { for } x=0
\end{array}\right.
$$

where $C_{p}$ is the cost of prototype testing and design. The function $\mathcal{H}$ is the Heaviside step function. We observe that: If all dampers are of the first size then $J_{p}$ will be equal to $C_{p} \times[1+0]$; If all dampers are of the second size then $J_{p}$ will be equal to $C_{p} \times[0+1]$; In case dampers of both sizes exist then $J_{p}$ will be equal to $C_{p} \times[1+1]$.

\subsection{Response constraints}

We are now considering the seismic retrofitting of 2-D hysteretic frames using nonlinear fluid viscous dampers. The retrofitting solution is achieved while considering constraints on the structural performance. In general, there are several local responses of interests related to the structural performance and damage such as inter-story drifts, total story accelerations, among others [16]. In what follows we will focus on inter-story drifts, as it has been shown that in many cases inter-story drifts are a good measure of both structural and non-structural damage [50]. In particular, the peak inter-story drift normalized 
by the allowable value is chosen as the local performance index for the structures considered:

$$
d_{c, i}=\max _{t}\left(\left|d_{i}(t) / d_{\text {allow }}\right|\right) \leq 1 \quad \forall i=1, \ldots, N_{\text {drifts }}
$$

where the inter-story drift $d_{i}(t)$ is the $i$-th constrained inter-story drift at time $t ; d_{\text {allow }}$ its maximum allowable value (e.g. $1 \%$ of the story height).

\subsection{Mixed-integer problem formulation}

The mixed-integer formulation of the optimization problem discussed herein that we would like to solve, is presented in (Prob1) (Sec. 4.2). For optimizing the distribution and size of a single damper size-group, only the $\mathbf{x}_{1}$ and $y_{1}$ variables are necessary, thus it can be seen as a particular case. The problem (Prob1) can be solved, for example, with a Genetic Algorithm (GA). The results obtained solving (Prob1) with a GA will be used for comparison in Sec. 5. It should be noted that in this work we consider a maximum allowable damping coefficient set a priori: $\bar{c}_{d}$. Moreover, since we consider dampers with a significant nonlinear viscous behavior, the maximum peak force that they can produce is indirectly constrained by their nonlinear mechanical behavior (defined by the exponent $\alpha$ in Eq. (5)) and by the predefined maximum available damping coefficient $\bar{c}_{d}$.

\section{Gradient-based optimization}

The computational effort required for the solution of the mixed-integer programming problem (Prob1) may be very high and in many cases prohibitive. Therefore, in the following we approximate (Prob1) with a continuous problem formulation that is solved with a lighter computational approach. Particular attention is given to achieving final practical (i.e. discrete) solutions. To this end, first we introduce the material interpolation functions used to promote the convergence towards final solutions characterized by crisp 0-1 distributions of the final values of the variables $x_{1, i}$ and $x_{2, i}$. Since the continuous problem is solved with a gradient-based algorithm, all the functions involved need to be differentiable. For this reason, we approximate two cost components and the constraints with differentiable approximations of the original formulations. We also reduce the number of constraints to one by aggregating them. This reduces the number of additional analyses required for the calculation of the constraints' gradients, or in other words the computational cost of the sensitivity analysis.

\subsection{Damping interpolation}

In the process of approximating (Prob1) with a continuous formulation we relax all the variables' definitions, so that in the final formulation they will all be continuous. As a consequence, the variables $\mathbf{x}_{1}$ and $\mathbf{x}_{2}$, which in principle were binary, now can assume the intermediate values between zero and one. These variables have a physical meaning only when they are equal to either zero or one, as was explained in Sec. 3.1. Therefore, we understand that their intermediate values should be avoided. Hence, we introduce in the problem formulation the so-called material interpolation functions. Their purpose is to penalize the intermediate values of the variables contained in the vectors $\mathbf{x}_{1}$ and $\mathbf{x}_{2}$ making them uneconomical, thus implicitly pushing the optimizer towards a preference of their boundary (i.e. 0,1 ) values.

These functions traditionally come from the field of structural topology and discrete material optimization. SIMP (Solid Isotropic Material with Penalization) is the most popular, and it proved to be very effective in a wide range of applications [51, 52]. Similarly, also RAMP (Rational Approximation of Material Properties) is a material interpolation function based on the same main idea [53]. Recently, the material interpolation functions have been introduced also in the context of optimization-based seismic retrofitting. Both SIMP and RAMP proved to be very effective for achieving final discrete solutions [15, 17, 36]. In the optimization problem presented herein, SIMP was chosen for the final problem formulation because it proved to be more effective and promising in leading the optimizer towards final discrete solutions. In this case, in fact, a combination of two SIMP functions has been applied to the vectors $\mathbf{x}_{1}$ and $\mathbf{x}_{2}$ in the damping formulation as follows [54, 55, 56, 57]:

$$
\tilde{c}_{d, i}=\bar{c}_{d} x_{1, i}^{p}\left(y_{1}+\left(y_{2}-y_{1}\right) x_{2, i}^{p}\right), \quad i=1, \ldots, 2 N_{d} .
$$


In Eq. (16) for $p=1$ we have a linear interpolation of the damping. For increasing values of $p$, the penalizing effect on the values of $\mathbf{x}_{1}$ and $\mathbf{x}_{2}$ between zero and one increases.

\subsection{Final problem formulation and computational considerations}

The continuous formulation of the optimization problem that we actually solve is presented in (Prob2), where for $\mathbf{x}_{1} \in \mathbb{R}^{2 N_{d}}, \mathbf{x}_{1}^{p}=\left[x_{1,1}^{p} \ldots x_{1,2 N_{d}}^{p}\right]^{T}$. The same definition applies to $\mathbf{x}_{2}^{p}$. The problem (Prob2 is highly nonlinear and nonconvex, and it has been solved with a modified Sequential Linear Programming approach (SLP) based on first order information. Hence, the cost components $J_{m}$ and $J_{p}$ have been approximated with differentiable formulations as in Pollini et al. [36. The formulation of the nonlinear constraints on the inter-story drifts (Eq. (15)) has also a non-differentiable function, $\max _{t}$. Thus, in (Prob2 the function $\max _{t}$ in Eq. (15) was approximated with a $r$-norm differentiable formulation (i.e. $\mathbf{d}_{c}$ ). Additionally, in order to reduce the number of sensitivity analyses from $N_{\text {drifts }}$ to one, we aggregated the inter-story drift constraints into a single constraint (i.e. $\tilde{d}_{c}$ ). More details regarding the constraint approximation and aggregation can be found in Reference[59] and Reference[36]. It should be noted that both $r$ and $q$ in (Prob2) are high numbers in order to obtain good approximations of the max function. The possibility of considering multiple ground acceleration records simultaneously has been discussed by the authors in Reference [17]. Here too, as we rely on a similar optimization approach, the methodology could accommodate multiple acceleration records, that could be seen as multiple load conditions. In what follows we discuss several aspects of the optimization algorithm.

$$
\begin{aligned}
& \min _{\mathbf{x}} J(\mathbf{x}) \\
& \begin{aligned}
\text { s.t.: } & d_{c, i}=\max _{t}\left(\left|\frac{d_{i}(t)}{d_{\text {allow }}}\right|\right) \leq 1, i=1, \ldots, N_{\text {drifts }} \\
& x_{1, j}=\{0,1\} \quad j=1, \ldots, 2 N_{d} \\
& x_{2, j}=\{0,1\} \quad j=1, \ldots, 2 N_{d} \\
& 0 \leq y_{1}<y_{2} \leq 1 \\
\text { with: } & \mathbf{M} \ddot{\mathbf{u}}(t)+\mathbf{C}_{s} \dot{\mathbf{u}}(t)+\mathbf{F}_{s}(t)+\mathbf{F}_{d}(t)=-\mathbf{M e} a_{g}(t) \\
& \mathbf{u}(0)=\mathbf{u}_{0}, \dot{\mathbf{u}}(0)=0, \mathbf{F}_{s}(0)=\mathbf{F}_{s, 0}, \mathbf{F}_{d}(0)=0 \\
& \mathbf{c}_{d}=\bar{c}_{d} \mathcal{D}\left(\mathbf{x}_{1}\right)\left(y_{1} \mathbf{1}+\left(y_{2}-y_{1}\right) \mathbf{x}_{2}\right), \mathbf{k}_{d}=\rho \mathbf{c}_{d} .
\end{aligned}
\end{aligned}
$$

$$
\begin{aligned}
\min _{\mathbf{x}} & \tilde{J}(\mathbf{x}) \\
\text { s.t.: } & \tilde{d}_{c}=\frac{\mathbf{1}^{T} \mathcal{D}^{q+1}\left(\tilde{\mathbf{d}}_{c}\right) \mathbf{1}}{\mathbf{1}^{T} \mathcal{D}^{q}\left(\tilde{\mathbf{d}}_{c}\right) \mathbf{1}} \leq 1 \\
& 0 \leq x_{1, j} \leq 1 \quad j=1, \ldots, 2 N_{d} \\
& 0 \leq x_{2, j} \leq 1 \quad j=1, \ldots, 2 N_{d} \\
& 0 \leq y_{1}<y_{2} \leq 1 \\
\text { with: } & \mathbf{M} \ddot{\mathbf{u}}(t)+\mathbf{C}_{s} \dot{\mathbf{u}}(t)+\mathbf{F}_{s}(t)+\mathbf{F}_{d}(t)=-\mathbf{M e} a_{g}(t) \\
& \mathbf{u}(0)=\mathbf{u}_{0}, \dot{\mathbf{u}}(0)=0, \mathbf{F}_{s}(0)=\mathbf{F}_{s, 0}, \mathbf{F}_{d}(0)=0 \\
& \tilde{\mathbf{c}}_{d}=\bar{c}_{d} \mathcal{D}\left(\mathbf{x}_{1}^{p}\right)\left(y_{1} \mathbf{1}+\left(y_{2}-y_{1}\right) \mathbf{x}_{2}^{p}\right), \tilde{\mathbf{k}}_{d}=\rho \tilde{\mathbf{c}}_{d} \\
& \tilde{\mathbf{d}}_{c}=\left(\frac{1}{t_{f}} \int_{0}^{t_{f}}\left(\mathcal{D}^{-1}\left(\mathbf{d}_{\text {allow }}\right) \mathcal{D}(\mathbf{H u}(t))\right)^{r} d t\right)^{\frac{1}{r}} \mathbf{1 .}
\end{aligned}
$$




\subsubsection{Sensitivity analysis}

The optimization procedure adopted for the solution of $(\overline{\text { Prob2 }})$ is iterative, and it solves a sequence of linearized approximations of the problem at hand. For this reason, the first-order derivatives of the objective function and of the aggregated inter-story drift constraint need to be calculated. The calculation of the objective function gradient in (Prob2) (i.e. $\left.\nabla_{\mathbf{x}} \tilde{J}\right)$ does not require any particular procedure, because it is formulated directly in terms of the variables of the problem. The gradient of the aggregated constraint (i.e. $\nabla_{\mathbf{x}} \tilde{d}_{c}$ ), on the other hand, requires an adjoint sensitivity analysis. To ensure the consistency of the sensitivity calculated, we relied on the discretize-then-differentiate adjoint variable method, similarly to Reference [45]. Ultimately, the outcome of adjoint sensitivity analysis is the vector $\nabla_{\mathbf{c}_{d}}^{T} \tilde{d}_{c}=\left[\begin{array}{lll}\frac{\partial \tilde{d}_{c}}{\partial c_{d 1}} & \cdots & \frac{\partial \tilde{d}_{c}}{\partial c_{d} N d}\end{array}\right]$. The complete derivatives are computed with the chain rule:

$$
\frac{d \tilde{d}_{c}}{d \mathbf{x}_{1}}=\frac{d \tilde{\mathbf{c}}_{d}}{d \mathbf{x}_{1}} \frac{\partial \tilde{d}_{c}}{\partial \tilde{\mathbf{c}}_{d}} ; \quad \frac{d \tilde{d}_{c}}{d \mathbf{x}_{2}}=\frac{d \tilde{\mathbf{c}}_{d}}{d \mathbf{x}_{2}} \frac{\partial \tilde{d}_{c}}{\partial \tilde{\mathbf{c}}_{d}} ; \quad \frac{d \tilde{d}_{c}}{d y_{1}}=\frac{d \tilde{\mathbf{c}}_{d}}{d y_{1}} \frac{\partial \tilde{d}_{c}}{\partial \tilde{\mathbf{c}}_{d}} ; \quad \frac{d \tilde{d}_{c}}{d y_{2}}=\frac{d \tilde{\mathbf{c}}_{d}}{d y_{2}} \frac{\partial \tilde{d}_{c}}{\partial \tilde{\mathbf{c}}_{d}} .
$$

\subsubsection{Continuation scheme}

The optimization problem $(\overline{\text { Prob2 }})$ is based on a few nonlinear components that increase its complexity, such as: the penalized damping coefficients, Eq. (16); the differentiable approximations of the max functions adopted for $\tilde{\mathbf{d}}_{c}$ and $\tilde{d}_{c}$ in $($ Prob2 $)$; the differentiable approximation of the Heaviside step function used in the reformulation of the third cost component $\tilde{J}_{p}[36$. These components are characterized by the parameters $p, s, r, q, \beta$, that need to assume high values in order to produce meaningful approximations. It is known that it is convenient to increase these parameters gradually in a stepwise manner, known also as a continuation scheme. In particular, the parameters $p$ and $s$ are increased by a fraction of their value every time a local convergence criterion in a continuation step is satisfied for a certain number of iterations. The criterion requires the norm of the variables' updates to be smaller than a certain tolerance for five consecutive iterations, that is: $\left\|\mathbf{x}_{k}-\mathbf{x}_{k-1}\right\| \leq \epsilon_{p, s}$. It was also observed that it is more convenient to start the optimization analysis with small but noticeable initial values of these parameters. The parameters $r$ and $q$ increase every iteration with a constant step-size; $\beta$, and the cost parameters $\mathbf{C}_{l}$ and $C_{p}$, are scaled by the coefficient $s$ which varies between zero and one. Moreover, conservative moving limits are imposed in the solution of the linear subproblems. As a consequence, the updates of the variables $\mathbf{x}_{1}, \mathbf{x}_{2}, y_{1}, y_{2}$, are found in a neighborhood of the solution of the previous subproblem. More details regarding these parameters are given in the numerical examples.

\subsubsection{Management of the constraints}

As it was already mentioned, (Prob2) was solved with a modified Sequential Linear Programming approach[19], in which in every iteration a linear subproblem is solved. Compared to traditional SLP approaches [60, 61, the algorithm discussed herein is a "modified" version because the subproblems expand, as in each iteration the new linearized constraint is added to the set of constraints considered. Due to the non-convexity of the problem at hand, and in particular of the aggregated constraint, it may happen that a constraint is active even though the current solution strictly falls into the feasible domain. In other words, it may happen that a constraint cuts the feasible domain directing the algorithm towards too conservative solutions. This is clearly shown in Fig. 4 of Reference [19]. The SLP algorithm considered in this work was modified in order to nullify and disregard these undesired constraints in the subsequent iterations.

\subsubsection{Convergence to a discrete solution}

The problem that we are discussing is a penalized continuous optimization problem. Therefore, initially for low values of the penalization the algorithm converges towards a continuous solution. Through the iterations, as the penalization (i.e. the parameter $p$ ) grows, the algorithm progressively converges towards solutions characterized by values of the variables $\mathbf{x}_{1}$ and $\mathbf{x}_{2}$ that approach their boundaries. However, during initial numerical experiments it proved to be quite challenging to converge to clean final discrete 
solutions, characterized by crisp distributions of $0-1$ values for the vectors $\mathbf{x}_{1}$ and $\mathbf{x}_{2}$. To improve this aspect, we modified the objective function to be minimized as in Reference [36]:

$$
J_{t o t}=\left(1-\frac{p}{p_{\max }}\right) \tilde{J}+\bar{c}_{d} \frac{p}{p_{\max }} J_{\text {binary }}
$$

where $J_{\text {binary }}$ is defined as follows [62, 63]:

$$
J_{\text {binary }}=\mathbf{x}_{1}^{T}\left(\mathbf{1}-\mathbf{x}_{1}\right)+\mathbf{x}_{2}^{T}\left(\mathbf{1}-\mathbf{x}_{2}\right)+y_{1}+y_{2}
$$

For given values of $y_{1}$ and $y_{2}$, the function $J_{\text {binary }}$ is at its minimum when all the values of $\mathbf{x}_{1}$ and $\mathbf{x}_{2}$ are binary. For intermediate values of $\mathbf{x}_{1}$ and $\mathbf{x}_{2}, J_{\text {binary }}$ is different from zero and positive. $J_{\text {binary }}$ is also multiplied by $\bar{c}_{d}$ so that numerically the two components of $J_{t o t}$ have comparable values. The purpose of $J_{\text {binary }}$ is to assist the material interpolation functions (Eq. (16)) in driving the algorithm towards final discrete solutions. In fact, for small values of the penalization $p$, the function to be minimized is $J_{t o t} \cong \tilde{J}$. As a consequence, the algorithm is driven towards initial minimum-cost continuous design solutions. When the penalization grows, the intermediate values of $\mathbf{x}_{1}$ and $\mathbf{x}_{2}$ become uneconomical: the algorithm starts to identify solutions more discrete. Simultaneously, the influence of the component $J_{\text {binary }}$ on the total cost increases, while the effect of $\tilde{J}$ decreases, so for high values of $p$ : $J_{\text {tot }} \cong J_{\text {binary }}$. The algorithm is pushed towards a preference of final solutions that are binary with respect to $\mathbf{x}_{1}$ and $\mathbf{x}_{2}$. This allows the translation of the initial continuous damping distribution into the closest mixed-integer solution.

\subsubsection{Convergence criteria}

The optimization process is assumed to have reached the final optimized solution if: The parameters $p$ and $s$ are equal to their maximum predefined values, that is $p_{\max }$ and $s_{\max }$; The norm of the update of the design variables in the current $k$-th iteration is smaller than a predefined value $\left\|\mathbf{x}_{k}-\mathbf{x}_{k-1}\right\|_{2} \leq \epsilon$; The maximum normalized inter-story drift $\tilde{d}_{c}$ is smaller than or equal to the allowable one, $d_{\text {allow }}$, with a certain tolerance. More details regarding these parameters will be given in Sec.5.

\section{$5 \quad$ Numerical examples}

In the following section, we present and discuss three numerical applications: the seismic retrofitting of a two-story frame, a nine-story frame, and a twenty-story frame. In the nine-story frame, we predefined nine potential locations for dampers in the central bay. In the twenty-story frame, we did not predefine any number of potential locations for dampers, allowing the algorithm to place dampers in all the 100 locations identified by story-bay intersections. The result is nine "cells" where a total of 18 dampers could be placed in the nine-story frame (two dampers per location), and 100 "cells" where a total of 200 dampers could be placed in the twenty-story frame (also two dampers per location). The continuous problem formulation (Prob2) was solved with a SLP approach, that was implemented in Matlab by the authors. The nine-story frame was optimized also by solving the mixed-integer problem formulation (Prob1) by using Matlab's built-in GA. The results achieved with the two formulations are used to compare the final dampers' distributions, and the computational effort required by the optimization analyses.

\subsection{Two-story frame: An illustrative example}

The first numerical application that we discuss is a two-story frame that was first considered by Lavan 64 for the seismic retrofitting with linear viscous dampers, Fig. 3(a). We believe that this example allows a better understanding of the approach discussed herein, since it allows a more clear graphic representation of the problem. The frame is subject to the ground acceleration record SE03 from the ensemble SE $10 \%$ in 50 years [65. In particular, we considered the first 10 seconds of the record. The bay width is $5.0 \mathrm{~m}$, and the story height is $3.0 \mathrm{~m}$. The frame has 12 degrees of freedom: two translations and one rotation for each node. The bending stiffness of the columns is $E I=45000 \mathrm{kN} / \mathrm{m}^{2}$, and the beams' bending stiffness is $E I=90000 \mathrm{kN} / \mathrm{m}^{2}$. Axial deformations in this case are neglected. The yielding moment of 


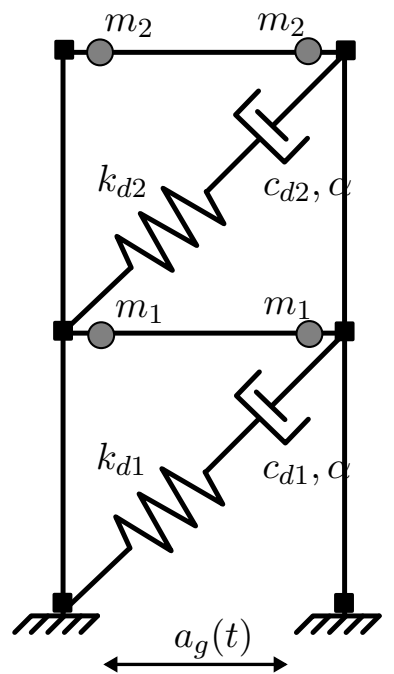

(a) Scheme of the two-story frame considered in Sec. 5.1 The variables are $c_{d 1}\left(x_{11}, y_{1}\right)$ and $c_{d 2}\left(x_{12}, y_{1}\right)$, for a given value of $\rho$ and $\alpha$.

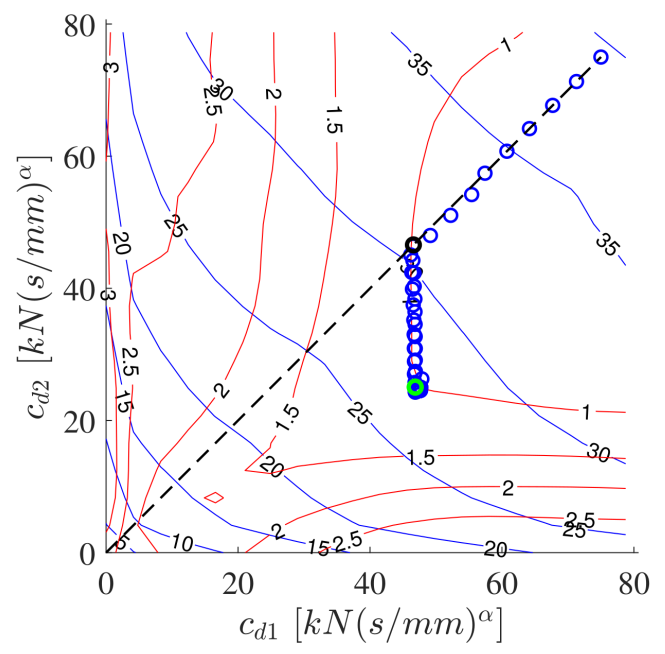

(b) Contour of the cost component $\tilde{J}_{m}$ (blue) and of the aggregated constraint (red) in the example of Sec. 5.1 Each blue circle represent the optimal solution of each sub-linear programming problem; the green circle represents the optimal solution of the continuous problem for low values of the penalization $p$; the black circle represent the final optimized solution

Figure 3: Two-story frame with two potential dampers and one dampers' size-group available

the columns is $M_{y}=63.5 \mathrm{kNm}$, the yielding moment in the lower beam is $M_{y}=72.0 \mathrm{kNm}$, and the yielding moment in the upper beam is $M_{y}=40.7 \mathrm{kNm}$. We considered $5 \%$ of critical damping for the first and second modes, whose periods are $0.3547 \mathrm{sec}$ and $0.1124 \mathrm{sec}$ respectively, in order to build the Rayleigh damping matrix of the structure. We considered a total mass for each story equal to 27 ton, so that $m_{1}=m_{2}=13.5$ ton. The exponent $\alpha$ of the dampers was set to 0.35 , and the ratio $\rho$ was set to 1 . We considered a maximum allowable inter-story drift equal to $d_{\text {allow }}=0.9 \mathrm{~cm}$.

The retrofitting problem was solved considering only its continuous formulation, i.e. (Prob2). In particular, we allowed as many as one damper in each location chosen from only one available size-group. Therefore, in this case the variables of the problem were only three: $\mathbf{x}_{1}=\left[x_{1,1} x_{1,2}\right]^{T}$ and $y_{1}$, and they were all initialized to 1 . The maximum allowable value for the inter-story drifts was set to $d_{\text {allow }}=0.9$ $\mathrm{cm}$; the maximum damping value available was set to $\bar{c}_{d}=75 \mathrm{kN}\left(\frac{\mathrm{s}}{\mathrm{mm}}\right)^{\alpha}$. It should be noted that only the first and second cost components are considered in this application, and that the vector with the cost parameters of the first cost component in this example was $\mathbf{C}_{l}=C_{l}\left[\begin{array}{ll}1 & 1\end{array}\right]^{T}$, with $C_{l}=25$ and $C_{m}=1$ $k N^{-0.5}$. With regards to the continuation scheme of the SLP algorithm: the parameter $p$ was increased from 1 to 3 by steps of 0.05 ; the parameter $s$ was increased from 0.5 to 1 by steps of 0.01 ; the parameters $r$ and $q$ were initialized to $10^{3}$ and increased indefinitely by steps of 10 .

Fig. 3(b) represents the design domain, in terms of $c_{d 1}$ and $c_{d 2}$. It shows also the contour of the second component of the objective function (i.e. $\tilde{J}_{m}$ ) in blue, and the contour of the aggregated normalized constraint in red. Each blue circle represents the optimized solution of each sub-linear programming problem. The black circle represents the final optimized solution, achived after 155 iterations. It is possible to observe that initially the algorithm converges towards the global optimal solution of the continuous problem, which is represented by the green circle. As the optimization progresses, and the penalization of the SIMP functions increases, the algorithm is pushed towards a preference of crisp $0-1$ final values of the variables $x_{1,1}$ and $x_{1,2}$ resulting in a discrete final solution: $c_{d 1}=c_{d 2}=46.58$ $k N\left(\frac{s}{m m}\right)^{\alpha}$. In correspondence of the final optimized solution, the objective function was $\tilde{J}=81.19$ and a small constraint violation, of approximately $0.1 \%$ of $d_{\text {allow }}$ (i.e. $0.009 \mathrm{~mm}$ ), was observed. However, from an engineering point of view this type of constraint violation is negligible. 


\subsection{Nine-story frame with 18 potential dampers}

In this example, we consider the north-south moment resisting frame of a nine-story building. The detailed description of the structure considered can be found in Reference [66]. With this example, we would like to compare the solution achieved with the gradient-based approach discussed in this paper with the one achieved with a GA. The solutions are compared in terms of dampers distributions, and computational efforts. In this case, we defined nine potential locations for dampers as shown in Fig. 4 . allowing a total of eighteen dampers to be potentially placed. The technical details of the frame can be found in Refrence [66].

For this example, the coefficient $\rho$ of the damper-brace elements was defined according to the procedure presented in Reference [36]. However, after numerical tests it was noticed that assigning the same parameter $\rho$ to all the dampers did not give the desired results. The dampers placed at higher stories tended to behave more as spring elements due to the participation of higher modes in the vibration of the higher stories. In fact, the output response of the dampers placed at higher stories revealed that they were experiencing higher frequencies of vibration, compared to the dampers at the bottom stories. For this reason, we modified the dampers' tuning procedure, and we considered in the tuning the first three modes of vibration of the structure. In particular, we considered $\omega_{1}$ for the dampers in locations $1-3$, $\omega_{2}$ for the dampers in locations $4-6$, and $\omega_{3}$ for the dampers in locations $7-9$. Additionally, since the stories had different heights, different values of allowable inter-story drifts were considered for tuning. The allowable value for the inter-story drift of the first story above ground was set to $5.49 \mathrm{~cm}$, and for all the remaining stories it was set to $3.96 \mathrm{~cm}$. Thus, we assigned to the damper-brace elements four different values of the parameters $\rho$ depending on their location in the structure: $\rho_{1}=0.5690, \rho_{2-3}=0.5194$, $\rho_{4-6}=0.8838$, and $\rho_{7-9}=1.1389$. The subscripts identify the location to which each $\rho$ coefficient refers to (Fig. 4).

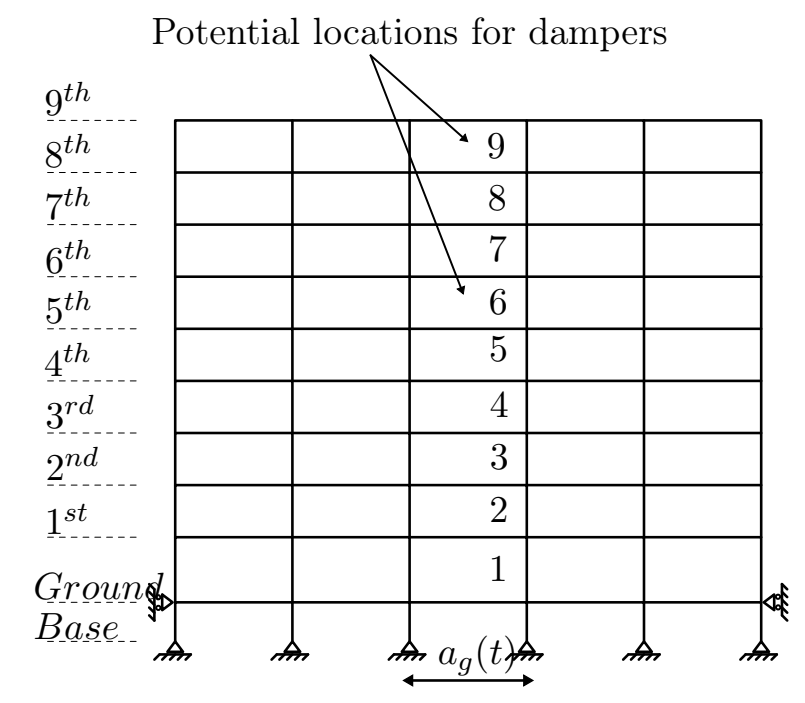

Figure 4: Potential locations for dampers in the nine-story moment resisting frame of Sec. 5.2

Regarding the ground motion acceleration, out of the ensemble $L A 10 \%$ in 50 years [67], LA05 was considered acting in the horizontal direction since it proved to cause the largest inter-story drift in the frame without dampers. In these examples, we considered $5 \%$ of critical damping for the first and the fifth modes in order to build the Rayleigh damping matrix of the structures. In Table 1 the numerical values of several parameters involved in the problem formulation are presented. It should be noted that the cost components different from zero of the vector $\mathbf{C}_{l 1}$ and $\mathbf{C}_{l 2}$ were all equal in the numerical experiments. Thus, with reference to Eq. (11), the non-zero components of $\mathbf{C}_{l 1}$ were equal to $C_{l 1}$, and similarly for $\mathbf{C}_{l 2}$ the non-zero components were equal to $C_{l 2}$. Moreover, the coefficient $\beta$ was used only in the SLP algorithm. In the GA, in fact, a true non-differentiable Heaviside step function was considered. Table2 lists the initial and final values, and the step sizes through which the parameters of the continuation scheme of the SLP algorithm are initialized and increased.

In the SLP algorithm we defined three criteria for convergence to be satisfied simultaneously: The first requires the parameters $p$, and $s$ to reach their maximum value; The second requires the update of 
Table 1: Values of the parameters used in the numerical examples of Sec. 5.2 ( $h$ is the story height)

\begin{tabular}{lcccccccc}
\hline Parameter & $d_{\text {allow }}$ & $\bar{c}_{d}$ & $\alpha$ & $C_{l 1}$ & $C_{l 2}$ & $C_{m}$ & $C_{p}$ & $\beta$ \\
& {$[$ as $h]$} & {$\left[k N\left(\frac{s}{m m}\right)^{\alpha}\right]$} & {$[\#]$} & {$[\#]$} & {$[\#]$} & {$\left[1 / k N^{0.5}\right]$} & {$[\#]$} & {$[\#]$} \\
\hline Value & $\frac{h}{100}$ & 500 & 0.35 & 100 & 50 & 1 & 50 & 100 \\
\hline
\end{tabular}

Table 2: Values of the parameters of the continuation scheme of the SLP algorithm in Sec. 5.2

\begin{tabular}{lrrrr}
\hline Parameter & $p$ & $s$ & $r$ & $q$ \\
\hline Initial value & 1.5 & 0.1 & 100 & 100 \\
Increment & $0.25 p$ & $0.25 s$ & 10 & 10 \\
Final value & 100 & 1 & $\infty$ & $\infty$ \\
\hline
\end{tabular}

the variables $\mathbf{x}_{k}-\mathbf{x}_{k-1}$ between two consecutive iterations to be smaller or equal to the $15 \%$ of $\mathbf{x}_{k-1}$; The third requires all the actual drifts to be smaller than the allowable value with a tolerance of $5 \%$.

In the GA implementation, the population size was set to 350 . In order to guarantee the convergence of the algorithm to a global optimum with high probability, five different analyses were performed, of which the best solution was chosen. In this case we defined two criteria for convergence: The first halts the algorithm when the number of generations (i.e. iterations) reaches the maximum number allowable Generations - 500; The second halts the algorithm when the weighted average relative change in the best fitness function value over StallGenLimit generations is less than or equal to TolFun. StallGenLimit is an integer set to 50 , and TolFun is a positive scalar set to $10^{-6}$.

In the SLP solution, a move limit of 0.1 was considered. The process converged after 159 iterations (approximately 3 hours and 20 minutes on a PC with a quad-core Intel processor at $3.60 \mathrm{GHz}$ ). Only one damping coefficient was actually assigned to the existing dampers. The optimized value for the damping coefficient was $\bar{c}_{2}=378.05 \mathrm{kN}\left(\frac{\mathrm{s}}{\mathrm{mm}}\right)^{\alpha}$. There was a small constraint violation in correspondence of the optimized solution at the first story (i.e. the second potential location for dampers), and the maximum drift was above the allowable value by $0.21 \%$ (i.e. $0.008 \mathrm{~cm}$ ). With GA, the analysis that led to the best solution converged after 73 iterations (approximately 4 days for the single GA analysis using 12 CPUs on a computer cluster hosted and maintained by the Division for Computing and Information Systems at the Technion - Israel Institute of Technology [68]), leading to a final distribution of dampers characterized by only one damping coefficient: $\bar{c}_{2}=497.49 \mathrm{kN}\left(\frac{\mathrm{s}}{\mathrm{mm}}\right)^{\alpha}$. The optimized distribution and damper sizes obtained with the two algorithms are shown in Fig. $5(\mathrm{a})$, and in Fig.5(b).

Table 3 provides a comparison of the solutions achieved. It is possible to observe that the solutions achieved with the two methods are characterized by similar topologies (Fig.5(a) and Fig.5(b)], and a final cost higher by $7.86 \%$ in the case of the SLP solution. In fact the two algorithms chose to distribute the dampers in the same locations. The solutions differ in the optimized sizes of the dampers' groups and in the total damping added in each location. This was already observed by the authors in previous work [17, 36], and it can be justified by the high non-convexity of the problem that causes the presence of several local minima in proximity of the global optima. The main advantage in solving this optimization problem with a gradient-based approach is the significant reduction in computational effort and time needed to achieve a good solution, compared to that of a GA. In fact, SLP required a number of timehistory analyses smaller by 2.605 orders of magnitude compared to that of GA. To get a satisfying solution with a GA we needed to consider a big population and to repeat several times the optimization analysis. In this case we considered a population of 350 individuals, meaning that the algorithm performed 350 time-history analyses in each iteration, and we repeated the optimization process 5 times. On the other hand, the SLP needed to compute two time-history analyses each iteration for just one optimization process: one for the structural response (nonlinear) and one for the evaluation of the constraint gradient (linear).

We performed an additional optimization analysis, this time considering a linear elastic behavior of the structure while optimizing nonlinear dampers. The goal was to check whether a retrofitting design solution obtained while considering a linear elastic structural behavior would have been appropriate also in the case of a nonlinear hysteretic behavior of the same structure. In this case, we solved the retrofitting problem only with the gradient-based approach (i.e. SLP). In the SLP algorithm, the maximum allowable damping 

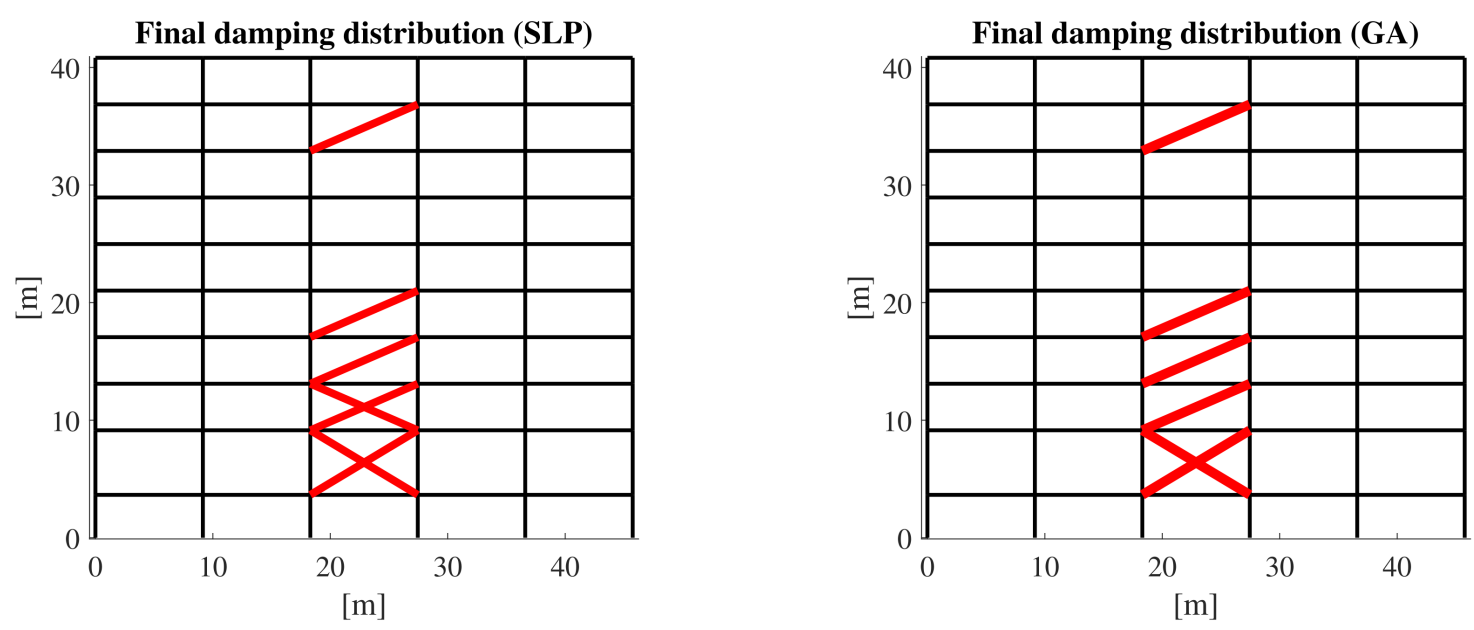

(a) SLP - Final damping distribution having 9 potential (b) GA - Final damping distribution having 9 potential locations for dampers (Sec. 5.2. Compared to GA, the so- locations for dampers (Sec. 5.2). Compared to SLP, the lution obtained with SLP requires one additional damper. solution obtained with GA requires one less damper. Only Only one size-group of dampers is actually present in the one size-group of dampers is actually present in the solusolution: $\bar{c}_{2}=378.05\left(k N\left(\frac{s}{m m}\right)^{\alpha}\right) \quad$ tion: $\bar{c}_{2}=497.49\left(k N\left(\frac{s}{m m}\right)^{\alpha}\right)$

Figure 5: Nine-story frame: optimized dampers distributions achieved with SLP and GA

Table 3: Solutions achieved in Ex.5.2 considering the record LA05. For the nonlinear structure, compared to GA SLP provides a solution with a similar cost, a small constraint violation, but with a computational effort smaller by 2.605 orders of magnitude

\begin{tabular}{lccccc}
\hline & $\begin{array}{c}\tilde{J}, J \\
{[\#]}\end{array}$ & $\frac{d_{c, \text { max }}}{d_{\text {allow }}}$ & $\begin{array}{c}\bar{c}_{1} \\
{\left[k N\left(\frac{s}{m m}\right)^{\alpha}\right]}\end{array}$ & $\begin{array}{c}\bar{c}_{2} \\
{\left[k N\left(\frac{s}{m m}\right)^{\alpha}\right]}\end{array}$ & $\begin{array}{c}\text { Time-history analyses } \\
(\text { Sensitivity analyses } \\
\text { are also counted in SLP) }\end{array}$ \\
\hline SLP (nonlinear structure) & 995.56 & $1.0006(0.06 \%)$ & $\mathrm{N} / \mathrm{A}$ & 391.08 & $2 \cdot 159 \approx 10^{2.502}$ \\
GA (nonlinear structure) & 923.04 & $1(0.00 \%)$ & $\mathrm{N} / \mathrm{A}$ & 497.49 & $5 \cdot 73 \cdot 350 \approx 10^{5.106}$ \\
SLP (linear structure) & 909.57 & $1.0015(0.15 \%)$ & $\mathrm{N} / \mathrm{A}$ & 451.26 & $2 \cdot 171 \approx 10^{2.534}$ \\
\hline
\end{tabular}

$\bar{c}_{d}$ was set $750 k N\left(\frac{s}{m m}\right)^{\alpha}$. The values of all the remaining parameters were identical to the previous example. The process converged after 169 iterations. The algorithm placed two dampers in location 1 , and one damper only in locations $2,3,6$, and 7 . Only one damping coefficient was actually assigned to the existing dampers: $\bar{c}_{2}=477.21 k N\left(\frac{s}{m m}\right)^{\alpha}$. Within the elastic behavior assumption, there was a small constraint violation at the ground story (corresponding to the first potential location for dampers) in correspondence of the optimized solution, and the maximum drift was above the allowable value by $0.09 \%$ (i.e. $0.005 \mathrm{~cm}$ ). In Table 3 we provide the details of the final optimized solution. The solution achieved considering a linear elastic behavior of the structure was then tested on the same structure, but allowing a hysteretic nonlinear structural behavior. In this case, a large constraint (i.e. inter-story drift) violation was observed, of approximately $13 \%$ of the allowable value. It is possible to conclude that for this specific design case it would not be safe to rely on a more simplified problem formulation (i.e. considering a linear elastic structural behavior) in order to identify an effective distribution of dampers. The structural behavior considered strongly influences the final optimized dampers' distribution, and its associated structural performance.

In the numerical applications discussed in this section we considered one level of seismic hazard associated to a predefined level of expected structural performance of the retrofitted structure. This was expressed in terms of a constraint on the maximum allowable inter-story drift, and for the seismic excitation considered only a limited amount of plasticity in the structure was expected. However, in Performance Based Seismic Design more severe levels of seismic excitation could be considered leading to larger deformation and plasticity demands. In these cases, dampers' distributions obtained under the assumption of linear structural behavior are expected to cause more significant drift constraints violations 


\section{Final damping distribution (SLP) (nonlinear structure)}

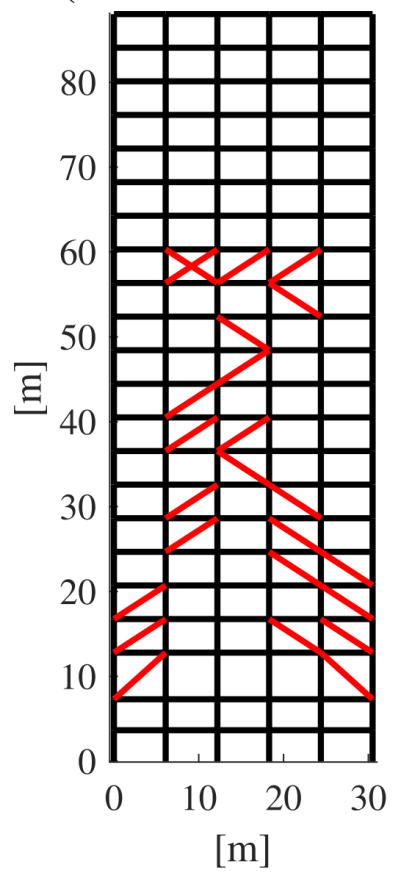

(a) SLP - Structure with nonlinear hysteretic behavior. Final damping distribution having 100 potential locations for dampers (Sec. 5.3) and allowing up to two dampers in each location chosen from only one available size-group $\left(\bar{c}_{1}=540.29\right.$ $\left.k N\left(\frac{s}{m m}\right)^{\alpha}\right)$

\section{Final damping distribution (SLP)}

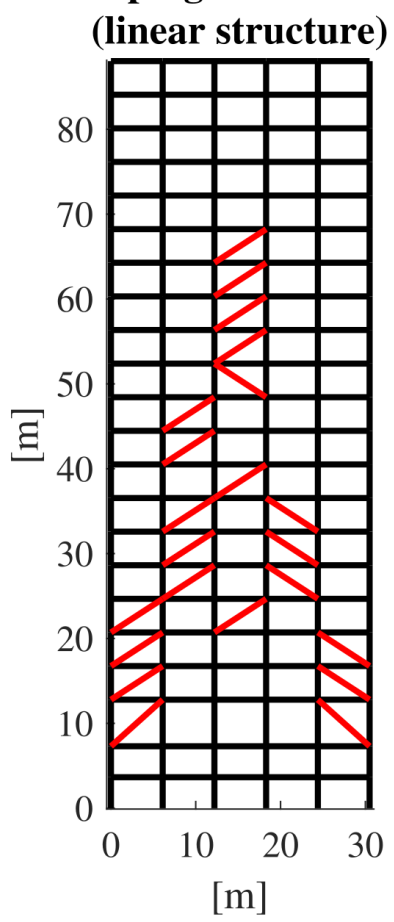

(b) SLP - Structure with linear behavior. Final damping distribution having 100 potential locations for dampers (Sec. 5.3) and allowing up to two dampers in each location chosen from only one available size-group $\left(\bar{c}_{1}=404.01 k N\left(\frac{s}{m m}\right)^{\alpha}\right)$

Figure 6: Twenty-story frame: optimized damper distributions achieved with SLP considering a nonlinear and linear structural behavior

if a nonlinear behavior is allowed.

\subsection{Twenty-story frame with 200 potential dampers}

The last example that we discuss concerns the retrofitting of a twenty-story moment resisting frame. The details of the structure considered can also be found in Reference [66]. With this example we would like to show the capability of the methodology discussed herein to handle large scale problems for the seismic retrofitting of tall buildings. We allowed the algorithm to place up to two dampers in all the five bays of each of the 20 stories of the building above ground, for a total of 100 potential locations for dampers and 200 dampers to be potentially placed. To reduce the combinatorial nature of the problem, we allowed the dampers to be chosen out of only one available size-group. Hence, only variables $\mathbf{x}_{1}$ and $y_{1}$ characterized the problem. Here too, as in Sec. 5.2, we relied on a modified tuning procedure for the parameter $\rho$ of the dampers. In fact, we considered the first five modes of the structure: $\omega_{1}$ for the dampers in stories $1-4, \omega_{2}$ for the dampers in stories $5-8, \omega_{3}$ for the dampers in locations $9-12, \omega_{4}$ for the dampers in locations $13-16$, and finally $\omega_{5}$ for the dampers in locations $17-20$. Due to the differences between the stories in terms of allowable inter-story drifts, six different values of $\rho$ were identified: $\rho_{1}=0.4383$, $\rho_{2-4}=0.4074, \rho_{5-8}=0.5857, \rho_{9-12}=0.7089, \rho_{13-16}=0.7988$, and $\rho_{17-20}=0.8786$. The subscripts define the story to which each $\rho$ coefficient refers to. Also in this example, the allowable value for the inter-story drift of the first story above ground was set to $5.49 \mathrm{~cm}$, and for all the remaining stories it was set to $3.96 \mathrm{~cm}$. The record $L A 04$ was selected out of the ensemble $L A 10 \%$ in 50 years [67], since it proved to cause the largest inter-story drift in the bare frame. Since only one size-group of dampers was considered in this example, the cost function was characterized by the first two components only: $\tilde{J}=J_{l}+\tilde{J}_{m}$. All the parameters' settings were identical to the example of Sec. 5.2 , the only differences 
being: $\bar{c}_{d}=1000 k N\left(\frac{s}{m m}\right)^{\alpha}$, the parameter $s$ initiated to 0.25 , and incremental steps of 50 in every iteration for the parameters $r$ and $q$.

This example was solved only with SLP, because GA failed to converge to final feasible optimized dampers' distributions. In the SLP algorithm, a more conservative moving limit of 0.025 was considered. The process converged after 180 iterations (approximately 16 hours and 24 minutes on a PC with a quad-core Intel processor at $3.60 \mathrm{GHz}$ ). The optimized value for the damping coefficient was $\bar{c}_{2}=540.29$ $k N\left(\frac{s}{m m}\right)^{\alpha}$. Fig. 6(a) shows the final dampers' distribution. It can be observed that the dampers are not distributed symmetrically in the structure. In fact, there are a few aspects that make the problem not symmetric: the ground motion is applied in one direction in Eq. (6), in the columns there is a initial axial force resulting from a preliminary static analysis, and we consider a nonlinear structural behavior. There was a small constraint violation in correspondence of the optimized solution at the fourth story above ground, and the maximum drift was above the allowable value by $0.05 \%$ (i.e. $0.002 \mathrm{~cm}$ ). Additionally, it was also observed that the axial forces at the bottom story in the structure with the optimized dampers' distribution were well within the elastic range.

In Table 4 we provide the details of the final optimized solution. An attempt to solve this problem using GA was made. In this case, the GA did not succeed in converging to a final optimized solution. The failure of the GA can probably be explained by the increased computational cost required for each response analysis of this large scale structure and by the increased combinatorial nature of the problem at hand. These two aspects together increase extremely the computational time required to converge to any satisfactory optimized solution. Additionally, a GA may produce intermediate designs characterized by dummy dampers' distributions and sizes remarkably unfeasible, especially in the initial generations (i.e. iterations). For these designs, the algorithm for the time-history analysis may be pushed to its limits in terms of converging to equilibrium points in every time-step. In large scale applications like the one described in this section, genetic algorithms are expected to required prohibitive computational costs and hence time, and in some cases they may even fail. In such cases, the only viable option may be to use more efficient gradient-based optimization methodologies, like the one discussed herein.

Table 4: Solutions in Sec. 5.3 considering the record $L A 04,100$ potential locations for dampers, and only one available size-group.

\begin{tabular}{lcccc}
\hline & $\tilde{J}$ & $\frac{d_{c, \max }}{d_{\text {allow }}}$ & $\begin{array}{c}\bar{c}_{1} \\
{\left[\# N\left(\frac{s}{m m}\right)^{\alpha}\right]}\end{array}$ & $\begin{array}{c}\text { Time-history analyses } \\
\text { (Sensitivity analyses } \\
\text { are also counted in SLP) }\end{array}$ \\
\hline SLP (nonlinear structure) & 3841.56 & $1.0005(0.05 \%)$ & 540.29 & $2 \cdot 180 \approx 10^{2.556}$ \\
SLP (linear structure) & 3338.58 & $1.0001(0.01 \%)$ & 404.01 & $2 \cdot 174 \approx 10^{2.542}$ \\
\hline
\end{tabular}

Also for this structure, we performed an additional optimization analysis considering a linear elastic structural behavior while optimizing nonlinear dampers. The goal was to check whether a retrofitting design solution obtained while considering a linear elastic structural behavior would have been appropriate also in the case of a nonlinear hysteretic behavior of the same structure. In this case, the solution obtained considering a linear elastic behavior (Fig. 6(b) was significantly different from the one obtained considering a nonlinear structural behavior (Fig. 6(a)b, in particular with respect to the dampers' number and distribution in the structure. The solution achieved considering a linear elastic behavior of the structure was then tested on the same structure, but allowing a hysteretic nonlinear structural behavior. In this case, a large constraint (i.e. inter-story drift) violation was observed, of approximately $40 \%$ of the allowable value. In particular, large plastic deformations were observed in several beams at the lower stories, and only a small amount of plasticity occurred in the columns of the ground floor. It is possible to conclude that also in this design case it would not be safe to rely on a more simplified problem formulation (i.e. considering a linear elastic structural behavior) in order to identify an effective distribution of dampers. The large constraint violation observed reveals the importance of the structural behavior considered in obtaining an effective optimized dampers' distribution. Additionally, it would not be trivial to define how the solution obtained considering a linear structural behavior should be modified so that it satisfies the constraint requirement when a nonlinear structural behavior is allowed. In fact, 
the two solutions (i.e. Fig. 6(a) and Fig. 6(b) are characterized by significantly different topologies and dampers' size.

\section{$6 \quad$ Final considerations}

In this paper, we discussed the problem of optimal seismic retrofitting of hysteretic frames with nonlinear fluid viscous dampers. The retrofitting approach consists of allocating and sizing nonlinear fluid viscous dampers and their supporting braces based on optimization. A realistic retrofitting cost function is minimized, considering the costs associated to the dampers' distribution in a structure, size, and prototype testing. The inter-story drifts are constrained to prescribed allowable values, collocating the approach discussed herein in the context of Performance Based Seismic Engineering. The responses of interest are computed with nonlinear time-history analyses considering realistic ground motions.

Major contributions of the approach presented herein are: the simultaneous nonlinear behavior of the dampers and of the structure. We consider also the axial force and bending moment interaction in the elements, and we allow the plasticity to spread along the beam-column elements; the formulation of a retrofitting problem that leads to final practical and realistic minimum-cost design solutions; the solution of the optimization problem with a computationally efficient gradient-based procedure. The approach presented in this paper is of particular interest in the case of seismic retrofitting for severe ground motions, especially for irregular and tall buildings, where the occurrence of plasticity in the structural elements and the effect of the dampers on the columns' axial forces at the lower stories need to be considered carefully. This represents the first application of this type, where the seismic retrofitting problem with viscous dampers is actually solved for hysteretic structures with nonlinear dampers in a gradient-based framework.

The numerical results showed how the proposed methodology successfully solved realistic design cases. We first discussed a simple application, that allows a more clear graphic understanding of the methodology adopted. Next, we applied the proposed approach to the seismic retrofitting of a realistic nine-story frame where both SLP and GA converged to similar final designs. With both algorithms, the solutions obtained should be considered as local minima, since with none of the algorithms considered it is possible to guarantee the convergence to the global minima of the problem. In both cases the designs were not intuitive, and significantly different from a more practical and intuitive solution where dampers would have been distributed along the full structure height with equal damping coefficients. If on the one hand the results achieved with the two algorithms were in good agreement in terms of structural performance and cost, on the other hand they also revealed a significant advantage of the gradient-based algorithm in terms of computational effort. The second and third applications also showed that, in some cases, optimizing the dampers assuming a linear behavior of the damped structure, may not be adequate. Last, we showed the capability of the approach to handle large scale applications, such as the seismic retrofitting of a twenty-story frame with potential dampers in all bays. This application represents a new contribution of this kind, and opens up new possibilities in the context of seismic retrofitting of nonlinear tall buildings. The potential of the approach presented became even more evident in the last numerical application, where the complexity of the problem formulation, and therefore of the analysis, significantly increased due to the simultaneous nonlinearity of the structure and of the dampers. The balance between the quality of the final solution and the effort required to achieve it, makes gradientbased approaches currently the only viable computational option for this type of applications. In many cases, in fact, practitioners dispose of limited computational resources and time for their realistic and complex applications, where genetic algorithms would require prohibitive computational efforts. In these cases, we expect that the proposed methodology will promote the optimization-based seismic retrofitting on nonlinear structures with fluid viscous dampers.

\section{Acknowledgments}

The authors are grateful to the anonymous reviewers for their helpful comments. The research presented in this paper was funded by the Israeli Ministry of Science, Technology and Space. The authors gratefully acknowledge this financial support. 


\section{References}

[1] PEER. Guidelines for performance-based seismic design of tall buildings - 2010/05. Pacific Earthquake Engineering Research Center, University of California Berkeley, 2010. (page 1)

[2] PEER. Modeling and acceptance criteria for seismic design and analysis of tall buildings - 2010/111. Pacific Earthquake Engineering Research Center, University of California Berkeley, 2010. (page 1)

[3] Wang S, Lai J-W, Schoettler M J, Mahin S A. Seismic assessment of existing tall buildings: A case study of a 35-story steel building with pre-Northridge connection. Engineering Structures. 2017; 141:624-633. (page 1)

[4] PEER. Seismic evaluation and retrofit of existing tall buildings in california. Pacific Earthquake Engineering Research Center, University of California Berkeley, California; 2015. (page 1)

[5] Wang S, Mahin S A. Seismic retrofit of a high-rise steel moment-resisting frame using fluid viscous dampers. The Structural Design of Tall and Special Buildings. 2017;26(10):e1367. (page 2)

[6] Soong T T, Dargush G F. Passive energy dissipation systems in structural engineering. Wiley New York; 1997. (page 2)

[7] Christopoulos C, Filiatrault A. Principles of passive supplemental damping and seismic isolation. IUSS Press; 2006. (page 2)

[8] Hahn G D, Sathiavageeswaran K R. Effects of added-damper distribution on the seismic response of buildings. Computers \& Structures. 1992;43(5):941-950. (page 2)

[9] Kanno Y. Damper placement optimization in a shear building model with discrete design variables: a mixed-integer second-order cone programming approach. Earthquake Engineering \& Structural Dynamics. 2013; 42(11):1657-1676. (page 2)

[10] Gluck N, Reinhorn A M, Gluck J, Levy R. Design of supplemental dampers for control of structures. Journal of Structural Engineering. 1996; 1394-1399. (page 2)

[11] Takewaki I. Optimal damper placement for minimum transfer functions. Earthquake Engineering 86 Structural Dynamics. 1997; 1113-1124. (page 2)

[12] Takewaki I. Optimal damper placement for planar building frames using transfer functions. Structural and Multidisciplinary Optimization. 2000; 20(4):280-287. (page 2)

[13] Singh M P, Moreschi L M. Optimal placement of dampers for passive response control. Earthquake Engineering and Structural Dynamics. 2002; 31:955-976. (page 2)

[14] Aydin E, Boduroglu M H, Guney D. Optimal damper distribution for seismic rehabilitation of planar building structures. Engineering Structures. 2007; 29(2):176-185. (page 2)

[15] Lavan O, Amir O. Simultaneous topology and sizing optimization of viscous dampers in seismic retrofitting of 3D irregular frame structures. Earthquake Engineering \& Structural Dynamics. 2014;43:1325-1342. (page 2, 9)

[16] Lavan O. Optimal design of viscous dampers and their supporting members for the seismic retrofitting of 3D irregular frame structures. Journal of Structural Engineering. 2015;141(11):04015026. (page 2, 8)

[17] Pollini N, Lavan O, Amir O. Towards realistic minimum-cost optimization of viscous fluid dampers for seismic retrofitting. Bulletin of Earthquake Engineering. 2016;14(3):971-998. (page 2, 7, 9, 10. $15)$

[18] Dargush G F, Sant R S. Evolutionary aseismic design and retrofit of structures with passive energy dissipation. Earthquake Engineering \& Structural Dynamics. 2005;34:1601-1626. (page 2) 
[19] Lavan O, Levy R. Optimal design of supplemental viscous dampers for irregular shear-frames in the presence of yielding. Earthquake Engineering 83 Structural Dynamics. 2005;34(8):889-907. (page 2 , 11)

[20] Levy R, Lavan O. Fully stressed design of passive controllers in framed structures for seismic loadings. Structural and Multidisciplinary Optimization. 2006;32(6):485-498. (page 2)

[21] Lavan O, Dargush G F. Multi-objective evolutionary seismic design with passive energy dissipation systems. Journal of Earthquake Engineering. 2009;13:758-790. (page 2)

[22] Lavan O, Levy R. Performance based optimal seismic retrofitting of yielding plane frames using added viscous damping. Earthquake and Structures. 2010;1(3):307-326. (page 2)

[23] Guo JWW, Christopoulos C. Performance spectra based method for the seismic design of structures equipped with passive supplemental damping systems . Earthquake Engineering 83 Structural Dynamics. 2012;42(6):935-952. (page 2)

[24] Aguirre J J, Almazán J L, Paul C J. Optimal control of linear and nonlinear asymmetric structures by means of passive energy dampers. Earthquake Engineering 83 Structural Dynamics. 2013;42(3):377395. (page 2)

[25] Shin H, Singh M P. Minimum failure cost-based energy dissipation system designs for buildings in three seismic regions - Part II: Application to viscous dampers. Engineering Structures. 2014;74:275282. (page 2)

[26] Akcelyan S, Lignos D G, Hikino T, Nakashima M. Evaluation of simplified and state-of-the-art analysis procedures for steel frame buildings equipped with supplemental damping devices based on E-Defense full-scale shake table tests. Journal of Structural Engineering. 2016;142(6):04016024. (page 2)

[27] Ji X, Hikino T, Kasai K, Nakashima M. Damping identification of a full-scale passively controlled fivestory steel building structure. Earthquake Engineering \& Structural Dynamics 2012, 42(2)pp.277295. (page 2)

[28] Tubaldi E, Barbato M, Dall'Asta A. Performance-based seismic risk assessment for buildings equipped with linear and nonlinear viscous dampers. Engineering Structures. 2014;78:90-99. (page2)

[29] Tubaldi E, Ragni L, Dall'Asta A. Probabilistic seismic response assessment of linear systems equipped with nonlinear viscous dampers. Earthquake Engineering \& Structural Dynamics. 2015;44(1):101-120. (page 2)

[30] Parcianello E, Chisari C, Amadio C. Optimal design of nonlinear viscous dampers for frame structures. Soil Dynamics and Earthquake Engineering 2017 100, pp.257-260. (page 2)

[31] Altieri D, Tubaldi E, De Angelis M, Patelli E, Dall'Asta A. Reliability-based optimal design of nonlinear viscous dampers for the seismic protection of structural systems. Bulletin of Earthquake Engineering 2018, pp.1-20. (page 2)

[32] Infanti S, Kang H T, Castellano M G. Retrofit of bridges in Korea using viscous damper technology. Proceedings of the 13th World Conference on Earthquake Engineering In: no. 2211; 2004. (page 2)

[33] Taylor Devices. Taylor Devices Inc. web page. (page 2)

[34] Lin W H, Chopra A K. Earthquake response of elastic SDF systems with non-linear fluid viscous dampers. Earthquake Engineering 85 Structural Dynamics. 2002;31:1623-1642. (page 2)

[35] Taylor D. Personal communication, 2015. (page 2, 8)

[36] Pollini N, Lavan O, Amir O. Minimum-cost optimization of nonlinear fluid viscous dampers and their supporting members for seismic retrofitting. Earthquake Engineering \& Structural Dynamics. 2017;46(12). (page 2, 3, 5, 7, 9, 10, 11, 12, 14, 15) 
[37] Gidaris I, Taflanidis A A. Performance assessment and optimization of fluid viscous dampers through life-cycle cost criteria and comparison to alternative design approaches. Bulletin of Earthquake Engineering. 2015;13(4):1003-1028. (page 2)

[38] Quagliaroli M. From bidimensional towards monodimensional modeling of sound and damaged reinforced concrete structures. PhD thesis, Politecnico di Milano, 2014. (page 3)

[39] Spacone E, Ciampi V, Filippou F C. A beam element for seismic damage analysis. Earthquake Engineering Research Center, College of Engineering, University of California Berkeley; 1992. (page 3 , 5. 6)

[40] Spacone E, Ciampi V, Filippou F C. Mixed formulation of nonlinear beam finite element. Computers E Structures. 1996; 58(I):71-83. (page 3)

[41] Sivaselvan M V, Reinhorn A M. Hysteretic models for deteriorating inelastic structures. Journal of Engineering Mechanics. 2000;126(6):633-640. (page 4)

[42] Carr A J. Ruaumoko manual. : University of Canterbury, Christchurch, New Zealand; 2007. (page 5)

[43] Kasai K, Oohara K. Algorithm and computer code to simulate response of nonlinear viscous dampers. Passively Controlled Structure Symposium, Yokohama, Japan, 2001 (page6)

[44] Akcelyan S, Lignos D G, Hikino T. Adaptive numerical method algorithms for nonlinear viscous and bilinear oil damper models subjected to dynamic loading. Soil Dynamics and Earthquake Engineering . 2018;113 (2018): 488-502.. (page 6)

[45] Pollini N, Lavan O, Amir O. Adjoint sensitivity analysis and optimization of hysteretic dynamic systems with nonlinear viscous dampers. Structural and Multidisciplinary Optimization. 2017;57(6):2273-2289. (page 6, 11)

[46] Chopra A K. Dynamics of Structures: Theory and Applications to Earthquake Engineering. PrenticeHall; 2007. (page )

[47] Puthanpurayil A M, Lavan O, Carr A J., Dhakal R P. Elemental damping formulation: an alternative modelling of inherent damping in nonlinear dynamic analysis. Bulletin of Earthquake Engineering. 2016;14(8):2405-2434. (page 6)

[48] Puthanpurayil A M, Lavan O, Dhakal R P. Seismic loss optimization of frame buildings using viscous dampers. Compdyn 2015. 2015. (page6)

[49] Quarteroni A, Sacco R, Saleri F. Numerical Mathematics Texts in Applied Mathematics, vol. 37: . Berlin, Heidelberg: Springer Berlin Heidelberg; 2007. (page 6)

[50] Charmpis D C, Komodromos P, Phocas M C. Optimized earthquake response of multi-storey buildings with seismic isolation at various elevations. Earthquake Engineering and Structural Dynamics. 2012;41(15):2289-2310. (page 8)

[51] Bendsøe M P, Sigmund O. Topology Optimization: Theory, Methods and Applications. Springer; 2003. (page 9)

[52] Eschenauer H A, Olhoff N. Topology optimization of continuum structures: A review*. Applied Mechanics Reviews. 2001;54(4):331-390. (page 9)

[53] Stolpe M, Svanberg K. An alternative interpolation scheme for minimum compliance topology optimization. Structural and Multidisciplinary Optimization. 2001;22:116-124. (page 9)

[54] Sigmund O, Torquato S. Design of materials with extreme thermal expansion using a three-phase topology. Journal of the Mechanics and Physics of Solids. 1997;45(6):1037-1067. (page 9)

[55] Stegmann J, Lund E. Discrete material optimization of general composite shell structures. International Journal for Numerical Methods in Engineering. 2005;62(May 2004):2009-2027. (page 9) 
[56] Hvejsel C F, Lund E. Material interpolation schemes for unified topology and multi-material optimization. Structural and Multidisciplinary Optimization. 2011;43(6):811-825. (page 9)

[57] Kanno Y. Global optimization of trusses with constraints on number of different cross-sections: a mixed-integer second-order cone programming approach. Computational Optimization and Applications. 2016;63(1):203-236. (page 9)

[58] Guest J K, Prévost J H, Belytschko T. Achieving minimum length scale in topology optimization using nodal design variables and projection functions. International Journal for Numerical Methods in Engineering. 2004;61(2):238-254. (page )

[59] Lavan O, Levy R. Optimal peripheral drift control of 3D irregular framed structures using supplemental viscous dampers. Journal of Earthquake Engineering. 2006;10(June 2013):903-923. (page 10)

[60] Kirsch U. Structural optimization. Springer; 1993. (page 11)

[61] Christensen P W, Klarbring A. An introduction to structural optimization. Springer; 2009. (page 11)

[62] Hvejsel C F, Lund E, Stolpe M. Optimization strategies for discrete multi-material stiffness optimization. Structural and Multidisciplinary Optimization. 2011;44(2):149-163. (page 12)

[63] Lipp T, Boyd S. Variations and extension of the convex-concave procedure. Optimization and Engineering. 2016;17(2):263-287. (page 12)

[64] Lavan O. Seismic behavior and control of irregular structures: Energy approach. PhD thesis, Technion - Israel Institute of Technology, 2006. (page 12)

[65] Somerville P, Smith N, Punyamurthula S, Sun J. Development of ground motion time history for phase 2 of the FEMA/SAC steel project. Report No. SAC/BD-97/04. : SAX/BD-97; 1997. (page 12)

[66] Ohtori Y, Christenson R E, Spencer B F, Dyke S J. Benchmark control problems for seismically excited nonlinear buildings. Journal of Engineering Mechanics. 2004;130(4):366-385. (page 14, 17)

[67] National Information Service for Earthquake Engineering - University of California Berkeley. 10 pairs of horizontal ground motions for Los Angeles with a probability of exceedence of $10 \%$ in 50 years. 2013. (page 14, 17)

[68] TAMNUN. Tamnun Cluster - High Performance Computing (HPC). (page 15 ) 